\title{
A mild $\mathrm{Cu}(\mathrm{I})$-catalyzed oxidative aromatization of indolines to indoles
}

Feng Peng, ${ }^{*}$ Mark McLaughlin, ${ }^{*}$ Yizhou Liu, Ian Mangion, David M. Tschaen, Yingju Xu

Department of Process Chemistry, Merck \& Co., Inc. Rahway, New Jersey 07065, USA

\section{Supporting Information}

Table of contents:

S2-S3: HPLC, ee, and PMI of the 500g indoline 3 oxidation demonstration.

S4-S7: ee of compound 9a, 9b, 9c, and 9d.

S8-S16: summary of high-through-put screening results and indoline 3 acid stress study.

S17-S19: summary of BHT and EPR study.

S20: Mechanism proposal for $\mathrm{Cu}(\mathrm{OAc})_{2} / \mathrm{TBHP}$ oxidation.

S21-S58: copies of ${ }^{1} \mathrm{H}$ and ${ }^{13} \mathrm{C}$ NMR spectra. 


\section{End of reaction slurry sample HPLC:}

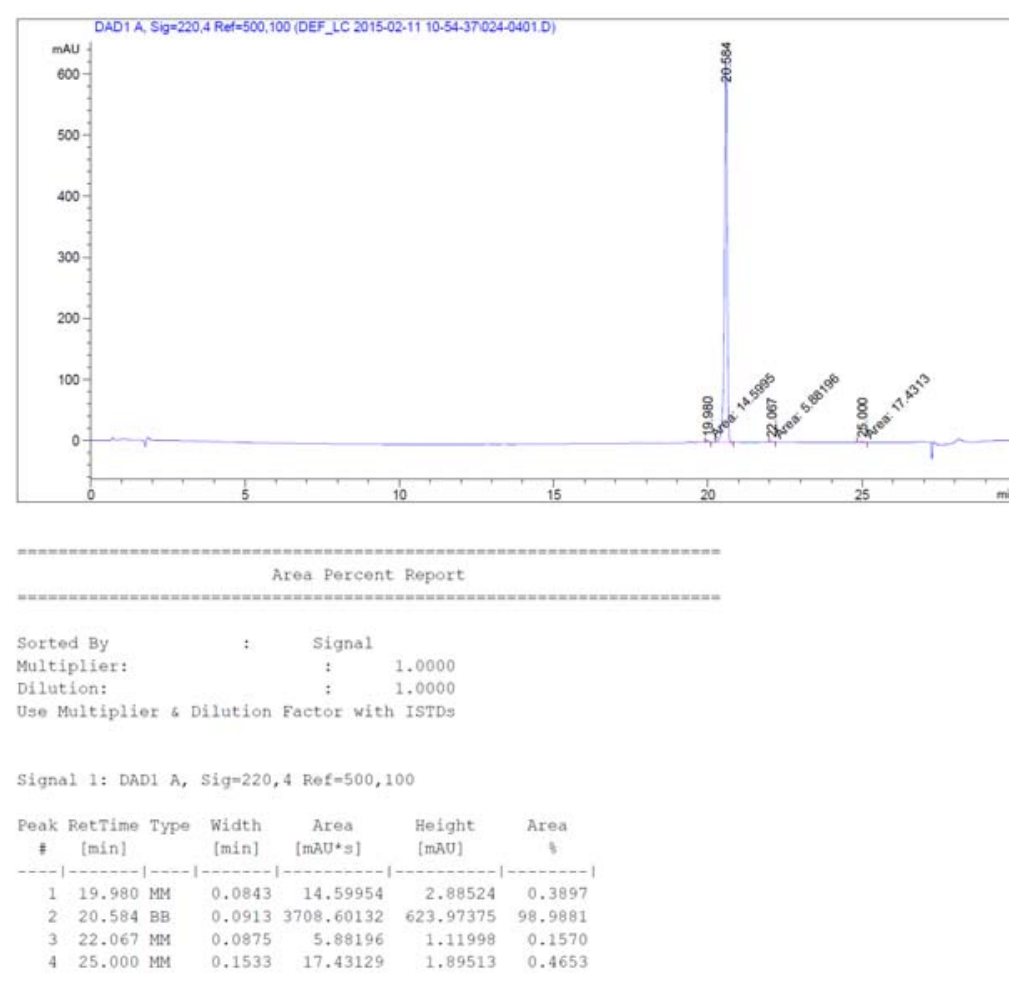

\section{Isolated indole 4 ee:}

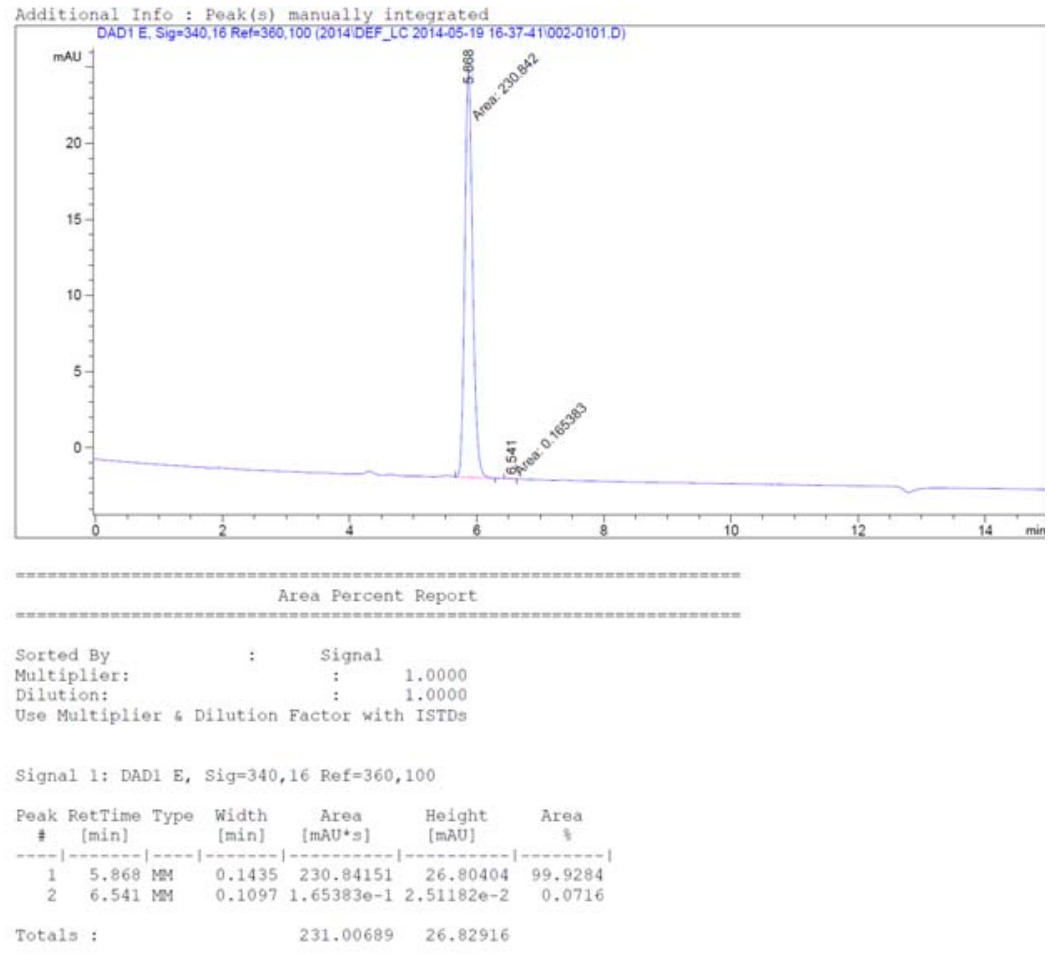


PMI comparison: $\mathrm{KMnO}_{4}$ process Vs Cu-catalyzed process:

\begin{tabular}{|l|c|c|}
\hline & $\mathrm{KMnO}_{4}$ & Cu-Oxidation \\
\hline Step Yield & $\mathbf{8 5 \%}$ & $\mathbf{9 2 \%}$ \\
\hline Step PMI Substrates, Reagents & $\mathbf{1 . 8}$ & $\mathbf{1 . 8}$ \\
\hline Step PMI Solvents & 33.2 & 6.7 \\
\hline Step PMI Water & $\mathbf{1 4 . 0}$ & $\mathbf{4 . 3}$ \\
\hline Total Step PMI & $\mathbf{4 9}$ & $\mathbf{1 2 . 8}$ \\
\hline
\end{tabular}

PMI = process mass intensity 


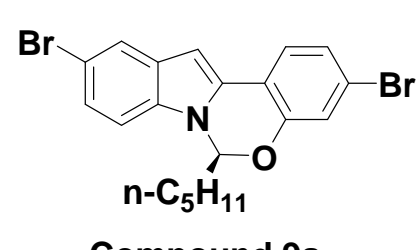

Compound 9a

$$
\text { eе }=99.2 \%
$$

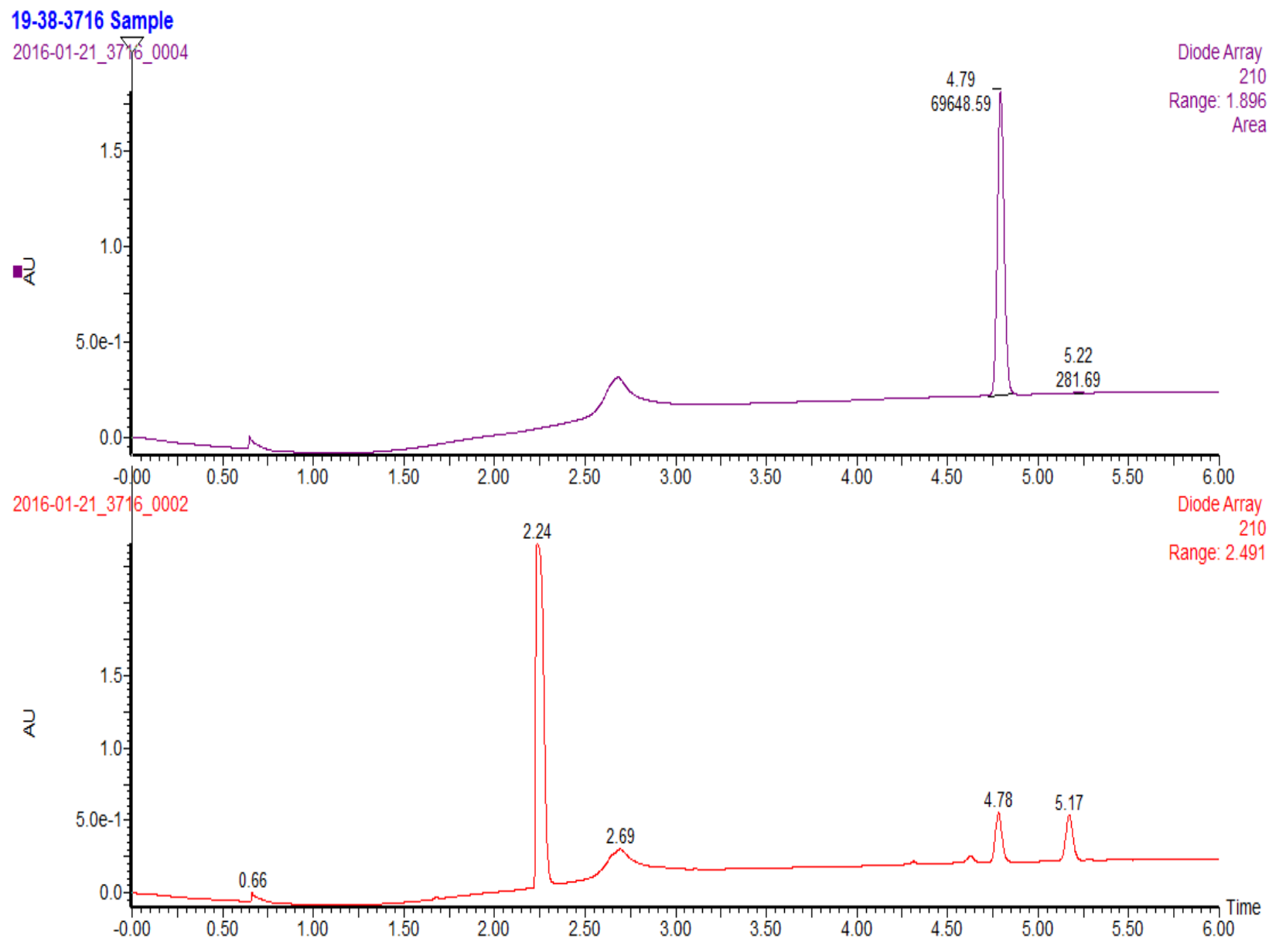




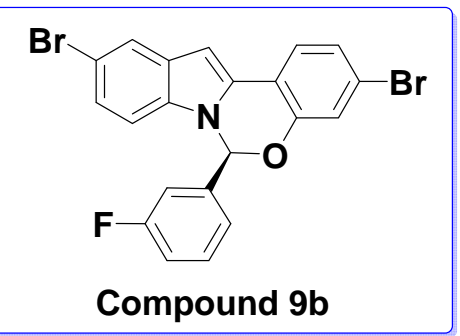

Racemic sample

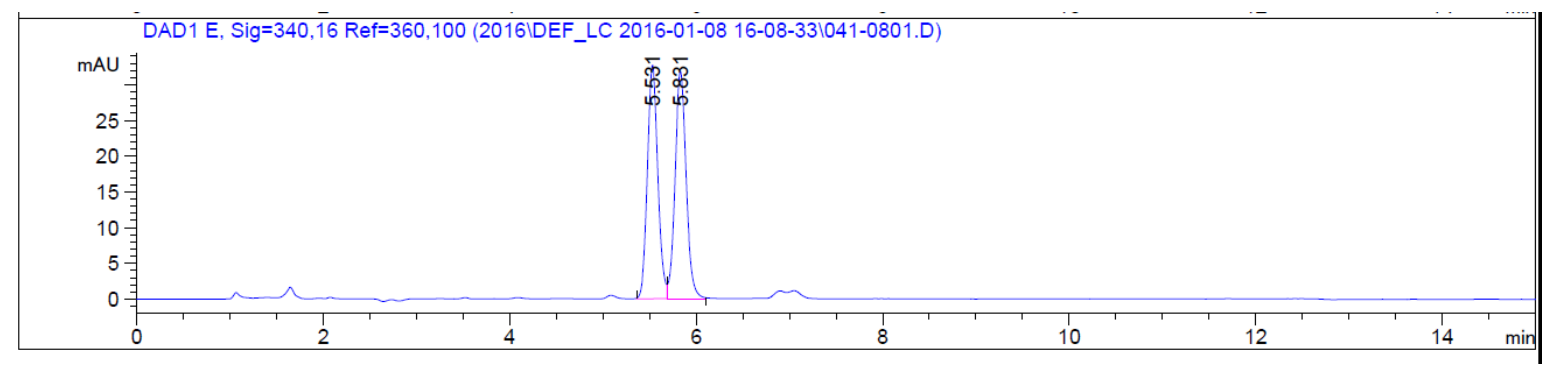

$\left[\mathrm{Cu}(\mathrm{MeCN})_{4}\right]\left(\mathrm{BF}_{4}\right)_{2} / \mathrm{TBPC}$ reaction

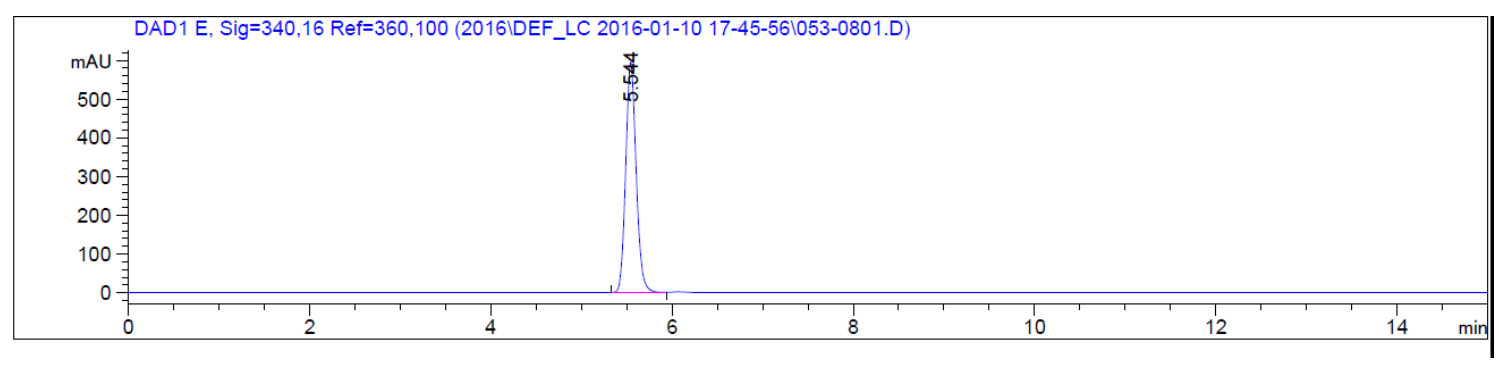

Signal 3: DAD1 E, Sig=340,16 Ref=360,100

\begin{tabular}{|c|c|c|c|c|c|c|}
\hline $\begin{array}{c}\text { Peak } \\
\quad \#\end{array}$ & $\begin{array}{c}\text { RetTime } \\
\text { [min] }\end{array}$ & Type & $\begin{array}{l}\text { Width } \\
\text { [min] }\end{array}$ & $\begin{array}{c}\text { Area } \\
{\left[\mathrm{mAU}^{*} \mathrm{~S}\right]}\end{array}$ & $\begin{array}{l}\text { Height } \\
\text { [mAU] }\end{array}$ & $\begin{array}{c}\text { Area } \\
\%\end{array}$ \\
\hline 1 & 5.544 & $\mathrm{BB}$ & 0.1236 & 4789.21875 & 596.76025 & 00.0000 \\
\hline
\end{tabular}

Totals :

$4789.21875 \quad 596.76025$ 


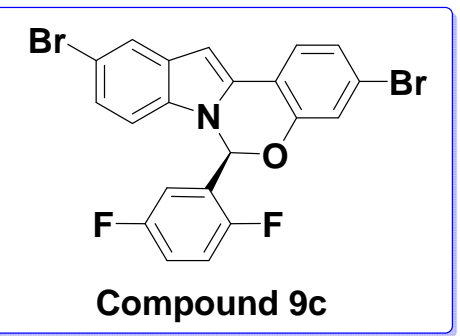

Racemic sample

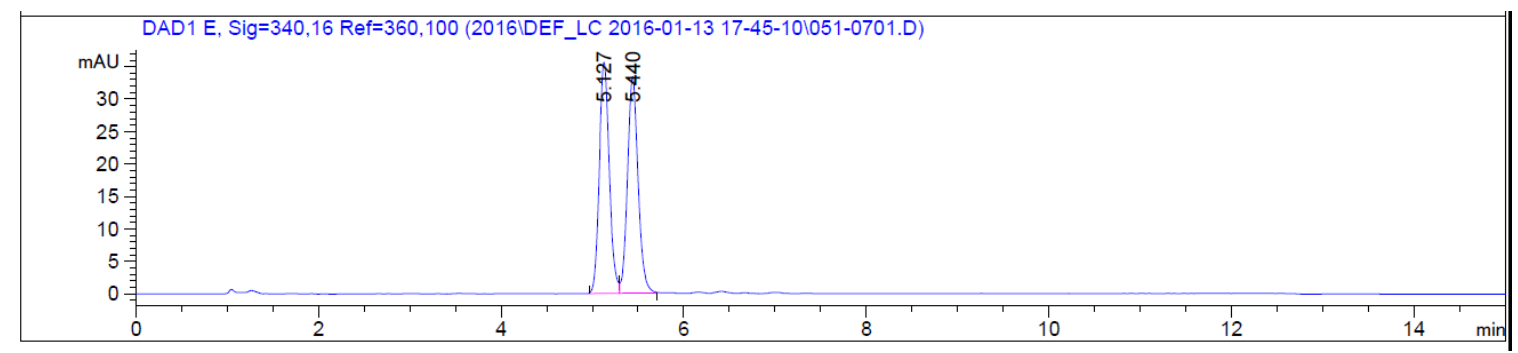

$\left[\mathrm{Cu}(\mathrm{MeCN})_{4}\right]\left(\mathrm{BF}_{4}\right)_{2} / \mathrm{TBPC}$ reaction

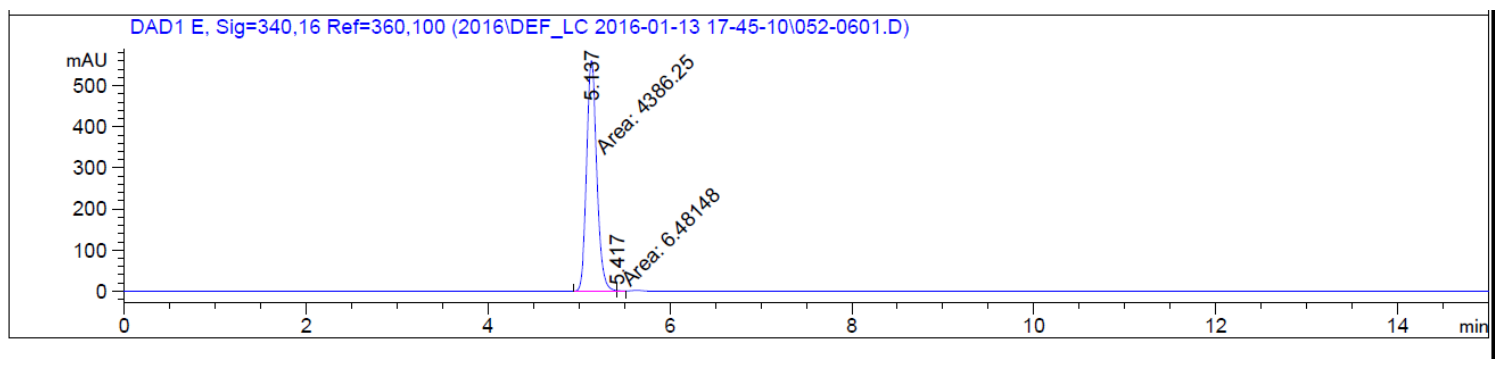

Signal 3: DAD1 E, Sig=340,16 Ref=360,100

\begin{tabular}{|c|c|c|c|c|c|c|}
\hline $\begin{array}{c}\text { Peak } \\
\#\end{array}$ & $\begin{array}{c}\text { RetTime } \\
\text { [min] }\end{array}$ & Type & $\begin{array}{c}\text { Width } \\
\text { [min] }\end{array}$ & $\begin{array}{c}\text { Area } \\
{\left[\mathrm{mAU}^{*} \mathrm{~S}\right]}\end{array}$ & $\begin{array}{l}\text { Height } \\
\text { [mAU] }\end{array}$ & $\begin{array}{c}\text { Area } \\
\frac{8}{8}\end{array}$ \\
\hline & & & & -------- & -------- & \\
\hline 1 & & $\mathrm{MF}$ & & 4386 . & 561.92432 & 99. \\
\hline 2 & 5.41 & M & & 6.48148 & .86053 & 476 \\
\hline
\end{tabular}

Totals : $\quad 4392.73002 \quad 563.78484$ 


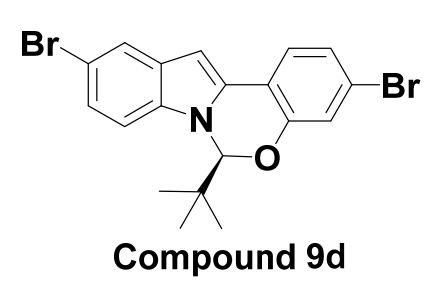

Racemic sample

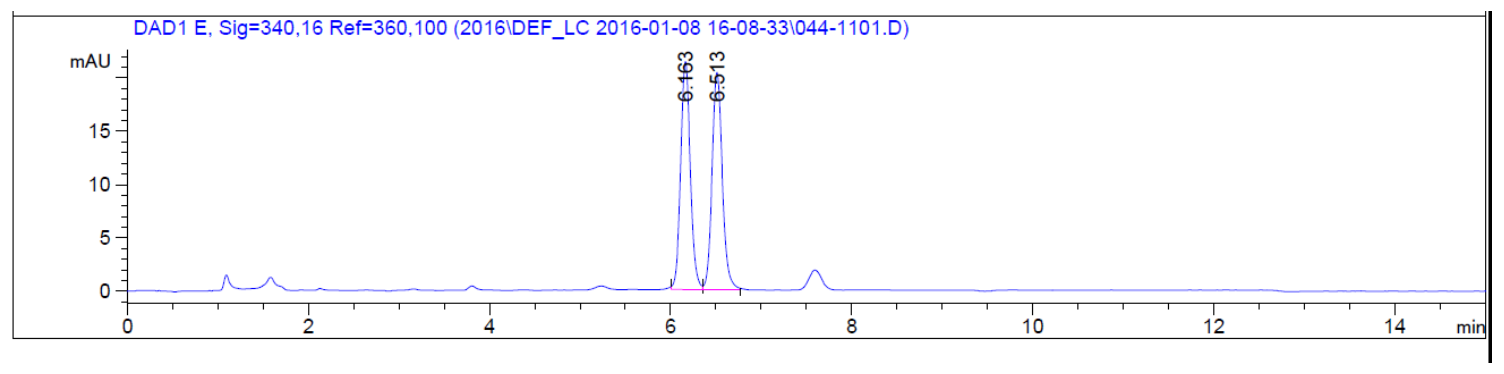

$\left[\mathrm{Cu}(\mathrm{MeCN})_{4}\right]\left(\mathrm{BF}_{4}\right)_{2} / \mathrm{TBPC}$ reaction

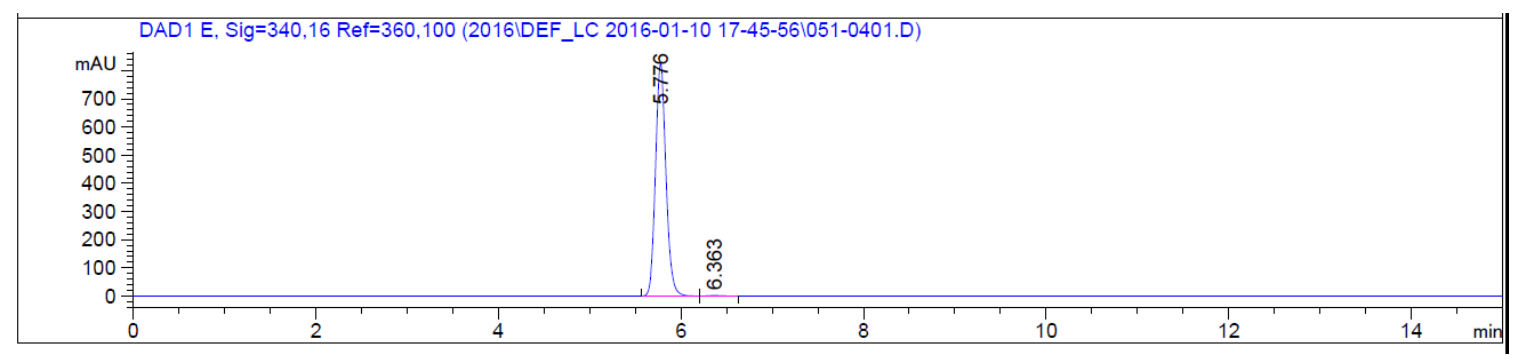

Signal 3: DAD1 E, Sig=340,16 Ref=360,100

\begin{tabular}{|c|c|c|c|c|c|c|}
\hline $\begin{array}{c}\text { Peak } \\
\#\end{array}$ & $\begin{array}{c}\text { RetTime } \\
\text { [min] }\end{array}$ & Type & $\begin{array}{l}\text { Width } \\
\text { [min] }\end{array}$ & $\begin{array}{c}\text { Area } \\
{\left[\mathrm{mAU}^{*} \mathrm{~s}\right]}\end{array}$ & $\begin{array}{l}\text { Height } \\
{[\mathrm{mAU}]}\end{array}$ & $\begin{array}{c}\text { Area } \\
\frac{\circ}{8}\end{array}$ \\
\hline & --1 & & & | --- & ---- & ----- \\
\hline 1 & 5.776 & $\mathrm{BB}$ & 0.1232 & 6594.30908 & 825.33997 & 99.6158 \\
\hline 2 & 6.363 & $\mathrm{BB}$ & 0.1665 & 25.43068 & 2.27308 & 0.3842 \\
\hline
\end{tabular}

Totals :

$$
6619.73976 \quad 827.61304
$$


Selected examples of 50 stoichoimetric indoline oxidation conditions screening:

\begin{tabular}{|c|c|c|c|c|}
\hline & $\mathrm{T} /{ }^{\circ} \mathrm{C}$ & Solvent & Conversion & LCAP \\
\hline TCNE & 80 & $\mathrm{DCM}$ & $90 \%$ & $50 \%$ \\
\hline $\mathrm{BzOOBz}$ & 80 & DMAC & $99 \%$ & $70 \%$ \\
\hline $\mathrm{K}_{2} \mathrm{~S}_{2} \mathrm{O}_{8}$ & 80 & DCM & $90 \%$ & $30 \%$ \\
\hline $\mathrm{LilO}_{3}$ & 80 & DCM & $90 \%$ & $80 \%$ \\
\hline $\mathrm{Phl}(\mathrm{OAc})_{2}$ & 80 & THF & $70 \%$ & $70 \%$ \\
\hline $\mathrm{AgNO}_{3}$ & 80 & THF & $100 \%$ & $40 \%$ \\
\hline $\mathrm{AgF}_{2}$ & 80 & DMAC & $100 \%$ & $90 \%$ \\
\hline $\mathrm{RuO}_{2}$ & 80 & $\mathrm{DCM}$ & $100 \%$ & $70 \%$ \\
\hline $\mathrm{WO}_{3}$ & 80 & THF & $100 \%$ & $10 \%$ \\
\hline $\mathrm{Fe}(\mathrm{acac})_{3}$ & 80 & DMAC & $70 \%$ & $10 \%$ \\
\hline $\mathrm{KMnO}_{4}$ & 40 & DMAC & $90 \%$ & $90 \%$ \\
\hline $\mathrm{KMnO}_{4}$ & 80 & DMAC & $100 \%$ & $95 \%$ \\
\hline $\mathrm{DDQ}$ & 80 & HOAc & $95 \%$ & $40 \%$ \\
\hline
\end{tabular}


Indoline substrate 3 Stress with Acids: pH Effect

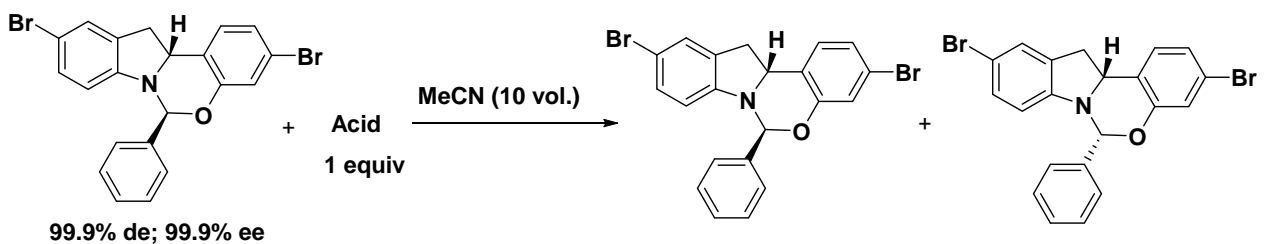

$99.9 \%$ de; $99.9 \%$ ee

\section{Benzoic Acid Acetic Acid}

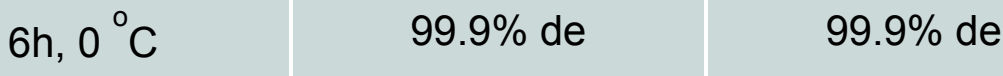

$6 h, r t$

$98.1 \%$ de

$99.2 \%$ de

$24 \mathrm{~h}, 0{ }^{\circ} \mathrm{C}$

$99.0 \%$ de

$99.6 \%$ de

$24 \mathrm{~h}, \mathrm{rt}$

$91.5 \%$ de

$95.7 \%$ de

Indoline substrate 3 Stress with metal catalysts: Lewis-Acid Effect

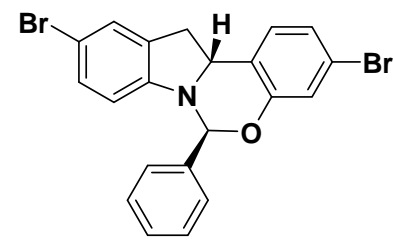

$99.9 \%$ de; $99.9 \%$ ee

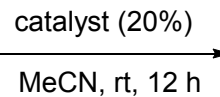

$\mathrm{MeCN}, \mathrm{rt}, 12 \mathrm{~h}$
$\mathrm{Br}$

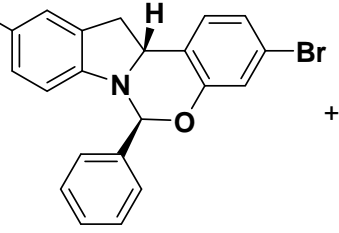

$\mathrm{Br}$

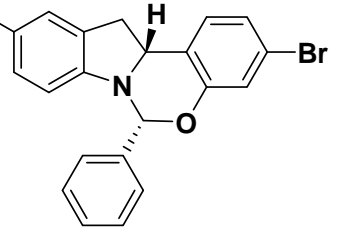

\begin{tabular}{|c|c|c|c|c|c|}
\hline Entry & Catalyst & de & Entry & Catalyst & de \\
\hline 1 & blank & $99.9 \%$ & 9 & $5 \% \mathrm{Ru} / \mathrm{C}$ & $87 \%$ \\
\hline 2 & {$\left[\mathrm{Cu}(\mathrm{MeCN})_{4}\right] \mathrm{BF}_{4}$} & $99.9 \%$ & 10 & $\mathrm{Mn}(\mathrm{OAc})_{2}$ & $96.4 \%$ \\
\hline 3 & {$\left[\mathrm{Cu}(\mathrm{MeCN})_{4}\right]\left(\mathrm{BF}_{4}\right)_{2}$} & Decomp. & 11 & $\mathrm{Fe}(\mathrm{acac})_{3}$ & $98.8 \%$ \\
\hline 4 & $5 \% \mathrm{Pd} / \mathrm{C}$ & $96 \%$ & 12 & $\mathrm{Cu}(\mathrm{TMHD})$ & $99.4 \%$ \\
\hline 5 & $10 \% \mathrm{Pd} / \mathrm{C}$ & $96 \%$ & 13 & $\mathrm{Cu}(\mathrm{OAc})_{2}$ & $99.2 \%$ \\
\hline 6 & $5 \% \mathrm{Pd} / \mathrm{Al}_{2} \mathrm{O}_{3}$ & $99 \%$ & 14 & $\mathrm{Cu}(\mathrm{OTf})_{2}$ & $78 \%$ \\
\hline 7 & $10 \% \mathrm{Pd} / \mathrm{Al}_{2} \mathrm{O}_{3}$ & $99.4 \%$ & 15 & $\mathrm{Ni}(\mathrm{OAc})_{2}$ & $99.6 \%$ \\
\hline 8 & $5 \% \mathrm{Pd} / \mathrm{CaCO}_{3}$ & $99.4 \%$ & & & \\
\hline
\end{tabular}


Summary of selected catalytic indoline oxidation conditions screened:
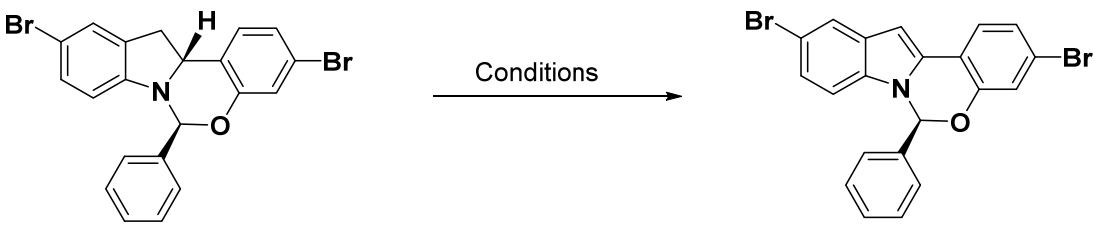

\begin{tabular}{|c|c|c|c|c|c|c|}
\hline Entry & Oxidant & Catalyst & Solvent & Additive & $\begin{array}{l}\text { Assay } \\
\text { Yield }\end{array}$ & ee \\
\hline 1 & TBHP & $\mathrm{Ni}(\mathrm{OAc})_{2}$ & $\mathrm{MeCN}$ & N/A & $12 \%$ & $42.5 \%$ \\
\hline 2 & TBHP & $\mathrm{Ni}(\mathrm{OAc})_{2} / \mathrm{by}$ & MeCN & N/A & $15 \%$ & $70.6 \%$ \\
\hline 3 & TBHP & $\mathrm{Ni}(\mathrm{OAc})_{2}$ & $\mathrm{MeCN}$ & $\mathrm{K}_{2} \mathrm{CO}_{3}$ & $7 \%$ & N/A \\
\hline 4 & TBHP & $\mathrm{Ni}(\mathrm{OAc})_{2} / \mathrm{by}$ & MeCN & $\mathrm{K}_{2} \mathrm{CO}_{3}$ & $9 \%$ & N/A \\
\hline 5 & TBHP & $\mathrm{Mn}(\mathrm{OAc})_{2}$ & MeCN & N/A & $70 \%$ & $93.1 \%$ \\
\hline 6 & TBHP & $\mathrm{Mn}(\mathrm{OAc})_{2} / \mathrm{by}$ & $\mathrm{MeCN}$ & N/A & $58 \%$ & $97.6 \%$ \\
\hline 7 & TBHP & $\mathrm{Mn}(\mathrm{OAc})_{2}$ & $\mathrm{MeCN}$ & $\mathrm{K}_{2} \mathrm{CO}_{3}$ & $40 \%$ & $96.3 \%$ \\
\hline 8 & TBHP & $\mathrm{Mn}(\mathrm{OAc})_{2} / \mathrm{by}$ & $\mathrm{MeCN}$ & $\mathrm{K}_{2} \mathrm{CO}_{3}$ & $50 \%$ & $98.1 \%$ \\
\hline 9 & TBHP & $\mathrm{Cu}(\mathrm{OAc})_{2}$ & $\mathrm{MeCN}$ & N/A & $65 \%$ & $99.1 \%$ \\
\hline 10 & TBHP & $\mathrm{Cu}(\mathrm{OAc})_{2} / \mathrm{by}$ & MeCN & N/A & $78 \%$ & $97.9 \%$ \\
\hline 11 & TBHP & $\mathrm{Cu}(\mathrm{OAc})_{2}$ & MeCN & $\mathrm{K}_{2} \mathrm{CO}_{3}$ & $79 \%$ & $97.6 \%$ \\
\hline 12 & TBHP & $\mathrm{Cu}(\mathrm{OAc})_{2} / \mathrm{by}$ & $\mathrm{MeCN}$ & $\mathrm{K}_{2} \mathrm{CO}_{3}$ & $76 \%$ & $99.5 \%$ \\
\hline 13 & TBHP & $5 \% \mathrm{Pd} / \mathrm{C}$ & MeCN & $\mathrm{K}_{2} \mathrm{CO}_{3}$ & $74 \%$ & $99.2 \%$ \\
\hline 14 & TBHP & $10 \% \mathrm{Pd} / \mathrm{C}$ & $\mathrm{MeCN}$ & $\mathrm{K}_{2} \mathrm{CO}_{3}$ & $63 \%$ & $98.9 \%$ \\
\hline 15 & TBHP & $10 \% \mathrm{Pd} / \mathrm{Al}_{2} \mathrm{O}_{3}$ & MeCN & $\mathrm{K}_{2} \mathrm{CO}_{3}$ & $51 \%$ & $98.7 \%$ \\
\hline 16 & TBHP & $5 \% \mathrm{Pd} / \mathrm{BaCO}_{3}$ & $\mathrm{MeCN}$ & $\mathrm{K}_{2} \mathrm{CO}_{3}$ & $36 \%$ & $99.2 \%$ \\
\hline 17 & TBHP & $5 \% \mathrm{Ru} / \mathrm{C}$ & MeCN & $\mathrm{K}_{2} \mathrm{CO}_{3}$ & $41 \%$ & $98.1 \%$ \\
\hline
\end{tabular}




\begin{tabular}{|c|c|c|c|c|c|c|}
\hline 18 & TBHP & C-941 & $\mathrm{MeCN}$ & $\mathrm{K}_{2} \mathrm{CO}_{3}$ & $32 \%$ & $97.3 \%$ \\
\hline 19 & TBHP & Aquarguard & $\mathrm{MeCN}$ & $\mathrm{K}_{2} \mathrm{CO}_{3}$ & $58 \%$ & $99.1 \%$ \\
\hline 20 & TBHP & Darco@KB & $\mathrm{MeCN}$ & $\mathrm{K}_{2} \mathrm{CO}_{3}$ & $40 \%$ & $98.7 \%$ \\
\hline 21 & TBHP & $\mathrm{CuCl}$ & $\mathrm{MeCN}$ & $\mathrm{K}_{2} \mathrm{CO}_{3}$ & $62 \%$ & $98.6 \%$ \\
\hline 22 & TBHP & $\mathrm{CuBr}$ & $\mathrm{MeCN}$ & $\mathrm{K}_{2} \mathrm{CO}_{3}$ & $61 \%$ & $98.1 \%$ \\
\hline 23 & TBHP & $\mathrm{CuI}$ & $\mathrm{MeCN}$ & $\mathrm{K}_{2} \mathrm{CO}_{3}$ & $75 \%$ & $98.9 \%$ \\
\hline 24 & TBHP & $\mathrm{Cu}(\mathrm{OPr})_{2}$ & $\mathrm{MeCN}$ & $\mathrm{K}_{2} \mathrm{CO}_{3}$ & $69 \%$ & $99.1 \%$ \\
\hline 25 & TBHP & $\mathrm{Cu}(\mathrm{OTs})_{2}$ & $\mathrm{MeCN}$ & $\mathrm{K}_{2} \mathrm{CO}_{3}$ & $71 \%$ & $90.2 \%$ \\
\hline 26 & TBHP & $\mathrm{Cu}(\mathrm{DMAA})_{2}$ & $\mathrm{MeCN}$ & $\mathrm{K}_{2} \mathrm{CO}_{3}$ & $3 \%$ & N/A \\
\hline 27 & TBHP & $\mathrm{Cu}(\text { PicolinicA })_{2}$ & $\mathrm{MeCN}$ & $\mathrm{K}_{2} \mathrm{CO}_{3}$ & $7 \%$ & N/A \\
\hline 28 & TBHP & $\mathrm{CuCl}_{2}$ & $\mathrm{MeCN}$ & $\mathrm{K}_{2} \mathrm{CO}_{3}$ & $62 \%$ & $98.6 \%$ \\
\hline 29 & TBHP & CuClOH(TMEDA) & $\mathrm{MeCN}$ & $\mathrm{K}_{2} \mathrm{CO}_{3}$ & $77 \%$ & $97.5 \%$ \\
\hline 30 & TBHP & $\mathrm{Cu}(\mathrm{dpm})_{2}$ & $\mathrm{MeCN}$ & $\mathrm{K}_{2} \mathrm{CO}_{3}$ & $3 \%$ & N/A \\
\hline 31 & TBHP & $\mathrm{Cu}$ (citric acid) & $\mathrm{MeCN}$ & $\mathrm{K}_{2} \mathrm{CO}_{3}$ & $3 \%$ & N/A \\
\hline 32 & TBHP & $\mathrm{Fe}(\text { acac })_{3}$ & $\mathrm{MeCN}$ & $\mathrm{K}_{2} \mathrm{CO}_{3}$ & $2 \%$ & N/A \\
\hline 33 & TBHP & $\mathrm{Mn}(\mathrm{dpm})_{2}$ & $\mathrm{MeCN}$ & $\mathrm{K}_{2} \mathrm{CO}_{3}$ & $74 \%$ & $98.5 \%$ \\
\hline 34 & TBHP & $\mathrm{I}_{2}$ & $\mathrm{MeCN}$ & $\mathrm{K}_{2} \mathrm{CO}_{3}$ & $2 \%$ & N/A \\
\hline 35 & TBHP & $\mathrm{Cu}(\mathrm{OAc})_{2}$ & $\mathrm{MeCN}$ & $\mathrm{K}_{2} \mathrm{CO}_{3}$ & $77 \%$ & $99.3 \%$ \\
\hline 36 & TBHP & CuOAc & $\mathrm{MeCN}$ & $\mathrm{K}_{2} \mathrm{CO}_{3}$ & $79 \%$ & $99.2 \%$ \\
\hline 37 & TBHP & $\mathrm{CuBr} \mathrm{Sme}_{2}$ & $\mathrm{MeCN}$ & $\mathrm{K}_{2} \mathrm{CO}_{3}$ & $57 \%$ & $98.6 \%$ \\
\hline 38 & TBHP & $\mathrm{Cu}(\mathrm{OBz})_{2}$ & $\mathrm{MeCN}$ & $\mathrm{K}_{2} \mathrm{CO}_{3}$ & $72 \%$ & $95.3 \%$ \\
\hline 39 & TBHP & $\mathrm{CuCN}$ & $\mathrm{MeCN}$ & $\mathrm{K}_{2} \mathrm{CO}_{3}$ & $61 \%$ & $99.0 \%$ \\
\hline 40 & TBHP & $\mathrm{FeBr}_{2}$ & $\mathrm{MeCN}$ & $\mathrm{K}_{2} \mathrm{CO}_{3}$ & $56 \%$ & $96.4 \%$ \\
\hline
\end{tabular}




\begin{tabular}{|c|c|c|c|c|c|c|}
\hline 41 & ТВHP & $\mathrm{Co}(\mathrm{OAc})_{2}$ & $\mathrm{MeCN}$ & $\mathrm{K}_{2} \mathrm{CO}_{3}$ & $70 \%$ & $97.8 \%$ \\
\hline 42 & ТВHР & $\mathrm{CoBr}_{2}$ & $\mathrm{MeCN}$ & $\mathrm{K}_{2} \mathrm{CO}_{3}$ & $3 \%$ & N/A \\
\hline 43 & TBHP & $\mathrm{CoCl}_{2}-6 \mathrm{H}_{2} \mathrm{O}$ & $\mathrm{MeCN}$ & $\mathrm{K}_{2} \mathrm{CO}_{3}$ & $72 \%$ & $98.8 \%$ \\
\hline 44 & TBHP & $\mathrm{Na}_{2} \mathrm{WO}_{4}-2 \mathrm{H}_{2} \mathrm{O}$ & $\mathrm{MeCN}$ & $\mathrm{K}_{2} \mathrm{CO}_{3}$ & $1 \%$ & N/A \\
\hline 45 & TBHP & $\mathrm{VO}(\text { асас })_{2}$ & $\mathrm{MeCN}$ & $\mathrm{K}_{2} \mathrm{CO}_{3}$ & $3 \%$ & N/A \\
\hline 46 & TBHP & $\mathrm{Re}_{2} \mathrm{O}_{7}$ & $\mathrm{MeCN}$ & $\mathrm{K}_{2} \mathrm{CO}_{3}$ & $3 \%$ & N/A \\
\hline 47 & TBHP & $\mathrm{RuCl}_{3}-\mathrm{H}_{2} \mathrm{O}$ & $\mathrm{MeCN}$ & $\mathrm{K}_{2} \mathrm{CO}_{3}$ & $32 \%$ & $97.5 \%$ \\
\hline 48 & ТВHP & $\mathrm{Cu}(\mathrm{OAc})_{2}$ & $\mathrm{MeCN}$ & $\mathrm{NaOBz}$ & $83 \%$ & $97.2 \%$ \\
\hline 49 & TBHP & $\mathrm{Cu}(\mathrm{OAc})_{2}$ & $\mathrm{MeCN}$ & $\mathrm{NBu}_{4} \mathrm{OBz}$ & $80 \%$ & $97.8 \%$ \\
\hline 50 & TBHP & $\mathrm{Cu}(\mathrm{OAc})_{2}$ & $\mathrm{MeCN}$ & $\mathrm{LiOBz}$ & $77 \%$ & $96.5 \%$ \\
\hline 51 & TBHP & $\mathrm{Cu}(\mathrm{OAc})_{2}$ & $\mathrm{MeCN}$ & pyridine & $68 \%$ & $98.7 \%$ \\
\hline 52 & TBHP & $\mathrm{Cu}(\mathrm{OAc})_{2}$ & $\mathrm{MeCN}$ & collidine & $81 \%$ & $99.3 \%$ \\
\hline 53 & TBHP & $\mathrm{Cu}(\mathrm{OAc})_{2}$ & $\mathrm{MeCN}$ & $\mathrm{NBu}_{4} \mathrm{OAc}$ & $83 \%$ & $98.1 \%$ \\
\hline 54 & $\mathrm{O}_{2}$ & $\mathrm{ZnI}_{2} /$ phd & $\mathrm{MeCN}$ & ppts & $<1 \%$ & N/A \\
\hline 55 & $\mathrm{O}_{2}$ & $\mathrm{Pd}(\mathrm{OAc})_{2}$ & $\mathrm{MeCN}$ & TEA/TEMPO & 0 & N/A \\
\hline 56 & $\mathrm{O}_{2}$ & $\mathrm{Ru}(\mathrm{OH}) \mathrm{x} / \mathrm{Al}_{2} \mathrm{O}_{3}$ & $\mathrm{MeCN}$ & N/A & 0 & N/A \\
\hline 57 & $\mathrm{O}_{2}$ & $\mathrm{CuCl}$ & $\mathrm{MeCN}$ & N/A & 0 & N/A \\
\hline 58 & $\mathrm{O}_{2}$ & $\begin{array}{c}{\left[\mathrm{Ru}(\mathrm{phd})_{3}\right]\left(\mathrm{PF}_{6}\right)_{2}} \\
\text { Co(salophen })\end{array}$ & $\mathrm{MeCN}$ & N/A & $<1 \%$ & N/A \\
\hline 59 & $\mathrm{H}_{2} \mathrm{O}_{2}$ & $\mathrm{Cu}(\mathrm{OAc})_{2}$ & MeCN & N/A & 0 & N/A \\
\hline 60 & $\mathrm{H}_{2} \mathrm{O}_{2}$ & $\mathrm{CuCl}$ & $\mathrm{MeCN}$ & N/A & 0 & N/A \\
\hline 61 & $\mathrm{H}_{2} \mathrm{O}_{2}$ & $\mathrm{CuBr}$ & $\mathrm{MeCN}$ & N/A & 0 & N/A \\
\hline 62 & $\mathrm{H}_{2} \mathrm{O}_{2}$ & $\mathrm{CuI}$ & $\mathrm{MeCN}$ & N/A & 0 & N/A \\
\hline
\end{tabular}




\begin{tabular}{|c|c|c|c|c|c|c|}
\hline 63 & $\mathrm{H}_{2} \mathrm{O}_{2}$ & $\mathrm{FeBr}_{2}$ & $\mathrm{MeCN}$ & $\mathrm{N} / \mathrm{A}$ & 0 & N/A \\
\hline 64 & $\mathrm{H}_{2} \mathrm{O}_{2}$ & $\mathrm{CoCl}_{2}$ & MeCN & N/A & 0 & N/A \\
\hline 65 & $\mathrm{H}_{2} \mathrm{O}_{2}$ & $\mathrm{~K}_{3}\left[\mathrm{Fe}(\mathrm{CN})_{6}\right]$ & $\mathrm{MeCN}$ & N/A & 0 & N/A \\
\hline 66 & $\mathrm{H}_{2} \mathrm{O}_{2}$ & $\mathrm{Co}(\mathrm{OAc})_{2}$ & $\mathrm{MeCN}$ & N/A & 0 & N/A \\
\hline 67 & $\mathrm{H}_{2} \mathrm{O}_{2}$ & $\mathrm{CoBr}_{2}$ & $\mathrm{MeCN}$ & N/A & 0 & N/A \\
\hline 68 & $\mathrm{H}_{2} \mathrm{O}_{2}$ & CuOAc & $\mathrm{MeCN}$ & N/A & 0 & N/A \\
\hline 69 & $\mathrm{H}_{2} \mathrm{O}_{2}$ & $\mathrm{CuCN}$ & $\mathrm{MeCN}$ & N/A & 0 & N/A \\
\hline 70 & air & $\mathrm{FeBr}_{2}$ & $\mathrm{MeCN}$ & TEMPO & 0 & N/A \\
\hline 71 & air & $\mathrm{Co}(\mathrm{OAc})_{2}$ & MeCN & TEMPO & 0 & N/A \\
\hline 72 & air & $\mathrm{CoBr}_{2}$ & $\mathrm{MeCN}$ & TEMPO & 0 & N/A \\
\hline 73 & air & $\mathrm{CoCl}_{2}$ & $\mathrm{MeCN}$ & TEMPO & 0 & N/A \\
\hline 74 & air & $\mathrm{CuCl}$ & MeCN & TEMPO & 0 & N/A \\
\hline 75 & air & $\mathrm{CuBr}$ & $\mathrm{MeCN}$ & TEMPO & 0 & N/A \\
\hline 76 & air & $\mathrm{CuI}$ & $\mathrm{MeCN}$ & TEMPO & 0 & N/A \\
\hline 77 & air & $\mathrm{Cu}(\mathrm{OAc})_{2}$ & $\mathrm{MeCN}$ & TEMPO & 0 & N/A \\
\hline 78 & air & CuClOH(TMEDA) & $\mathrm{MeCN}$ & TEMPO & $7 \%$ & N/A \\
\hline 79 & air & $\mathrm{Cu}(\mathrm{DMAA})_{2}$ & MeCN & TEMPO & $12 \%$ & N/A \\
\hline 80 & ТВPB & $\mathrm{CuCl}$ & $\mathrm{MeCN}$ & N/A & $72 \%$ & $86 \%$ \\
\hline 81 & ТВPB & $\mathrm{CuBr}$ & MeCN & N/A & $<5 \%$ & N/A \\
\hline 82 & ТВPB & $\mathrm{CuI}$ & MeCN & N/A & $<5 \%$ & N/A \\
\hline 83 & TBPB & $\mathrm{CuCN}$ & MeCN & N/A & $<5 \%$ & N/A \\
\hline 84 & TBPB & CuOAc & $\mathrm{MeCN}$ & N/A & $<5 \%$ & N/A \\
\hline 85 & ТВРB & $\mathrm{Cu}(\mathrm{OAc})_{2}$ & MeCN & N/A & $<5 \%$ & N/A \\
\hline
\end{tabular}




\begin{tabular}{|c|c|c|c|c|c|c|}
\hline 86 & TBPB & $\mathrm{Cu}(\mathrm{OTs})_{2}$ & $\mathrm{MeCN}$ & $\mathrm{N} / \mathrm{A}$ & $<5 \%$ & N/A \\
\hline 87 & TBPB & $\mathrm{Cu}(\mathrm{OPr})_{2}$ & $\mathrm{MeCN}$ & N/A & $15 \%$ & $88 \%$ \\
\hline 88 & ТВРВ & $\mathrm{Cu}(\mathrm{OBz})_{2}$ & $\mathrm{MeCN}$ & N/A & $<5 \%$ & N/A \\
\hline 89 & TBPB & $\mathrm{CuCl}_{2}$ & MeCN & N/A & $78 \%$ & $72 \%$ \\
\hline 90 & TBPB & $\mathrm{CuCl}(\mathrm{OH})(\mathrm{TMEDA})_{2}$ & $\mathrm{MeCN}$ & N/A & $<5 \%$ & N/A \\
\hline 91 & TBPB & DDQ & $\mathrm{MeCN}$ & N/A & $<5 \%$ & N/A \\
\hline 92 & TBPA & $\mathrm{CuBr}$ & $\mathrm{MeCN}$ & $\mathrm{N} / \mathrm{A}$ & $16 \%$ & $77 \%$ \\
\hline 93 & TBPA & $\mathrm{CuCl}$ & $\mathrm{MeCN}$ & $\mathrm{N} / \mathrm{A}$ & $79 \%$ & $90 \%$ \\
\hline 94 & TBPA & $\mathrm{CuI}$ & $\mathrm{MeCN}$ & N/A & $19 \%$ & $75 \%$ \\
\hline 95 & TBPA & $\mathrm{CuCN}$ & $\mathrm{MeCN}$ & N/A & $21 \%$ & $74 \%$ \\
\hline 96 & TBPA & CuOAc & $\mathrm{MeCN}$ & N/A & $28 \%$ & $82 \%$ \\
\hline 97 & TBPA & $\mathrm{Cu}(\mathrm{OAc}) 2$ & $\mathrm{MeCN}$ & N/A & $<5 \%$ & N/A \\
\hline 98 & TBPA & $\mathrm{Cu}(\mathrm{Ots}) 2$ & $\mathrm{MeCN}$ & N/A & $12 \%$ & N/A \\
\hline 99 & TBPA & $\mathrm{CuCl} 2$ & $\mathrm{MeCN}$ & N/A & $81 \%$ & $76 \%$ \\
\hline 100 & TBPA & $\mathrm{CuCl}(\mathrm{OH})(\mathrm{TMEDA}) 2$ & $\mathrm{MeCN}$ & N/A & $32 \%$ & $87 \%$ \\
\hline 101 & TBPC & $\mathrm{CuCl}$ & $\mathrm{MeCN}$ & N/A & $77 \%$ & $99.9 \%$ \\
\hline 102 & TBPC & $\mathrm{CuCl}_{2}$ & $\mathrm{MeCN}$ & N/A & $80 \%$ & $88.6 \%$ \\
\hline 103 & TBPC & $\mathrm{CuBr}$ & $\mathrm{MeCN}$ & N/A & $12 \%$ & N/A \\
\hline 104 & TBPC & $\mathrm{CuI}$ & $\mathrm{MeCN}$ & N/A & $35 \%$ & $96.8 \%$ \\
\hline 105 & TBPC & $\mathrm{CuCN}$ & $\mathrm{MeCN}$ & N/A & $26 \%$ & $97.8 \%$ \\
\hline 106 & TBPC & CuOAc & $\mathrm{MeCN}$ & N/A & $28 \%$ & $99.1 \%$ \\
\hline 107 & TBPC & $\mathrm{Cu}(\mathrm{OAc})_{2}$ & $\mathrm{MeCN}$ & N/A & $6 \%$ & N/A \\
\hline 108 & TBPC & $\mathrm{Cu}(\mathrm{OTs})_{2}$ & $\mathrm{MeCN}$ & N/A & $8 \%$ & N/A \\
\hline
\end{tabular}




\begin{tabular}{|c|c|c|c|c|c|c|}
\hline 109 & TBPC & $\mathrm{Cu}(\mathrm{OPr})_{2}$ & $\mathrm{MeCN}$ & N/A & $7 \%$ & N/A \\
\hline 110 & ТВРC & $\mathrm{Cu}(\mathrm{OBz})_{2}$ & $\mathrm{MeCN}$ & N/A & $12 \%$ & N/A \\
\hline 111 & TBPC & $\mathrm{Cu}(\mathrm{DMAA})_{2}$ & $\mathrm{MeCN}$ & N/A & $32 \%$ & $95.6 \%$ \\
\hline 112 & TBPC & $\mathrm{CuCl} / \mathrm{by}$ & MeCN & N/A & $81 \%$ & $99.9 \%$ \\
\hline 113 & TBPC & $\mathrm{CuCl}_{2} / \mathrm{by}$ & $\mathrm{MeCN}$ & N/A & $82 \%$ & $99.8 \%$ \\
\hline 114 & TBPC & $\mathrm{FeBr}_{2}$ & $\mathrm{MeCN}$ & N/A & $20 \%$ & $94.1 \%$ \\
\hline 115 & TBPC & $\mathrm{Co}(\mathrm{OAc})_{2}$ & $\mathrm{MeCN}$ & N/A & $31 \%$ & $99.1 \%$ \\
\hline 116 & TBPC & $\mathrm{Ni}(\mathrm{OAc})_{2}$ & $\mathrm{MeCN}$ & N/A & $23 \%$ & $98.2 \%$ \\
\hline 117 & TBPC & $\mathrm{FeCp}_{2}$ & $\mathrm{MeCN}$ & N/A & $<5 \%$ & N/A \\
\hline 118 & TBPC & $\mathrm{Fe}(\mathrm{OAc})_{2}$ & $\mathrm{MeCN}$ & N/A & $25 \%$ & $95.8 \%$ \\
\hline 119 & TBPC & {$\left[\mathrm{FeCp}_{2}\right]\left[\mathrm{BF}_{4}\right]$} & $\mathrm{MeCN}$ & N/A & $<5 \%$ & N/A \\
\hline 120 & TBPC & {$\left[\mathrm{FeCp}_{2}\right]\left[\mathrm{PF}_{6}\right]$} & $\mathrm{MeCN}$ & N/A & $<5 \%$ & N/A \\
\hline 121 & TBPC & $\mathrm{FeCl}_{2}$ & $\mathrm{MeCN}$ & $\mathrm{N} / \mathrm{A}$ & & \\
\hline 122 & TBPC & $\mathrm{FeO}$ & $\mathrm{MeCN}$ & N/A & $<5 \%$ & N/A \\
\hline 123 & TBPC & $\mathrm{FeMoO}_{4}$ & $\mathrm{MeCN}$ & N/A & $15 \%$ & N/A \\
\hline 124 & TBPC & $\mathrm{MnCl}_{2}$ & $\mathrm{MeCN}$ & N/A & $7 \%$ & N/A \\
\hline 125 & TBPC & $\mathrm{Mn}(\mathrm{OAc})_{2}$ & $\mathrm{MeCN}$ & N/A & $7 \%$ & N/A \\
\hline 126 & TBPC & $\mathrm{Mn}(\mathrm{OAc})_{3}$ & $\mathrm{MeCN}$ & N/A & $8 \%$ & N/A \\
\hline 127 & TBPC & {$\left[\mathrm{Cu}(\mathrm{MeCN})_{4}\right]\left[\mathrm{BF}_{4}\right]$} & $\mathrm{MeCN}$ & N/A & $91 \%$ & $99.9 \%$ \\
\hline 128 & TBPC & {$[\mathrm{Cu}(\mathrm{MeCN}) 4]\left[\mathrm{PF}_{6}\right]$} & $\mathrm{MeCN}$ & N/A & $82 \%$ & $99.9 \%$ \\
\hline 129 & TBPC & {$[\mathrm{Cu}(\mathrm{MeCN}) 4][\mathrm{BF} 4]$} & DMA & N/A & $22 \%$ & $99.9 \%$ \\
\hline 130 & TBPC & [Cu(MeCN)4][BF4] & DMF & N/A & $20 \%$ & $99.9 \%$ \\
\hline 131 & TBPC & {$[\mathrm{Cu}(\mathrm{MeCN}) 4][\mathrm{BF} 4]$} & Acetone & N/A & $53 \%$ & $98.2 \%$ \\
\hline
\end{tabular}




\begin{tabular}{|c|c|c|c|c|c|c|}
\hline 132 & TBPC & {$[\mathrm{Cu}(\mathrm{MeCN}) 4][\mathrm{BF} 4]$} & $\mathrm{Cl}$ & $\mathrm{N} / \mathrm{A}$ & $76 \%$ & $95.3 \%$ \\
& & & benzene & & \\
\hline 133 & TBPC & {$\left[\mathrm{Cu}(\mathrm{MeCN})_{4}\right]\left[\mathrm{BF}_{4}\right]$} & IPAc & $\mathrm{N} / \mathrm{A}$ & $35 \%$ & $99.3 \%$ \\
\hline 134 & TBHP & $\mathrm{Cu}(\mathrm{OAc}) 2$ & $\mathrm{DMA}$ & $\mathrm{K}_{2} \mathrm{CO}_{3}$ & $32 \%$ & $99.1 \%$ \\
\hline 135 & TBHP & $\mathrm{Cu}(\mathrm{OAc}) 2$ & $\mathrm{Cl}-$ & $\mathrm{K}_{2} \mathrm{CO}_{3}$ & $65 \%$ & $96.3 \%$ \\
& & & benzene & & & \\
\hline
\end{tabular}




\section{BHT experiment:}

To a $8 \mathrm{~mL}$ vial with a magnetic stir bar were charged indoline 3 (100 mg), acetonitrile (1 $\mathrm{mL}), \mathrm{BHT}(48 \mathrm{mg})$ and $\left[\mathrm{Cu}(\mathrm{MeCN})_{4}\right]\left(\mathrm{BF}_{4}\right)$ (0.05 mol equiv to indoline) under nitrogen. The reaction mixture was heated to $35{ }^{\circ} \mathrm{C}$ and charged with tert-Butylperoxy 2-ethylhexyl carbonate (TBPC, $1.2 \mathrm{~mol}$ equiv to indoline) dropwise. The reaction was aged at $35{ }^{\circ} \mathrm{C}$ for 4 hours. About 10\% conversion by HPLC assay.

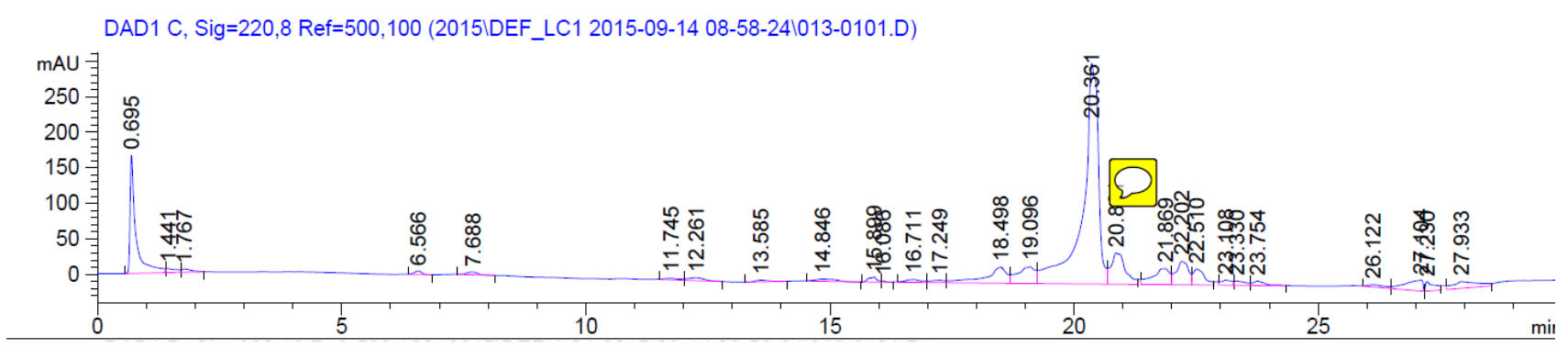

Indoline SM at $20.36 \mathrm{~min}$, indole product at $20.8 \mathrm{~min}$. 


\section{Experimental Procedure for Spin Trap of Proposed tert-Butoxy Radical for X-band}

\section{cw-EPR Observation}

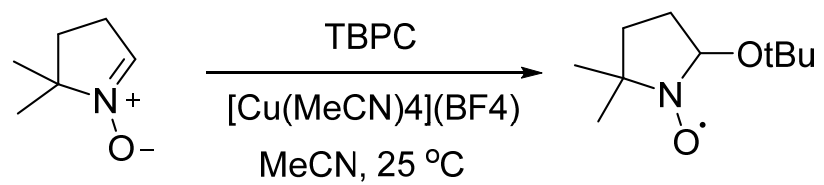

DMPO (5,5-Dimethyl-1-pyrroline N-oxide) was used as the spin trap molecule.

The EPR sample solution was made by mixing Solution A (60mM [Cu(MeCN)4](BF4) in MeCN) and Solution B (60mM TBPC and 60mM DMPO in MeCN) at 1:1 v/v ratio, and $40 \mathrm{uL}$ of the resulting mixture was quickly transferred to a glass capillary tube for reaction monitoring by EPR spectroscopy.

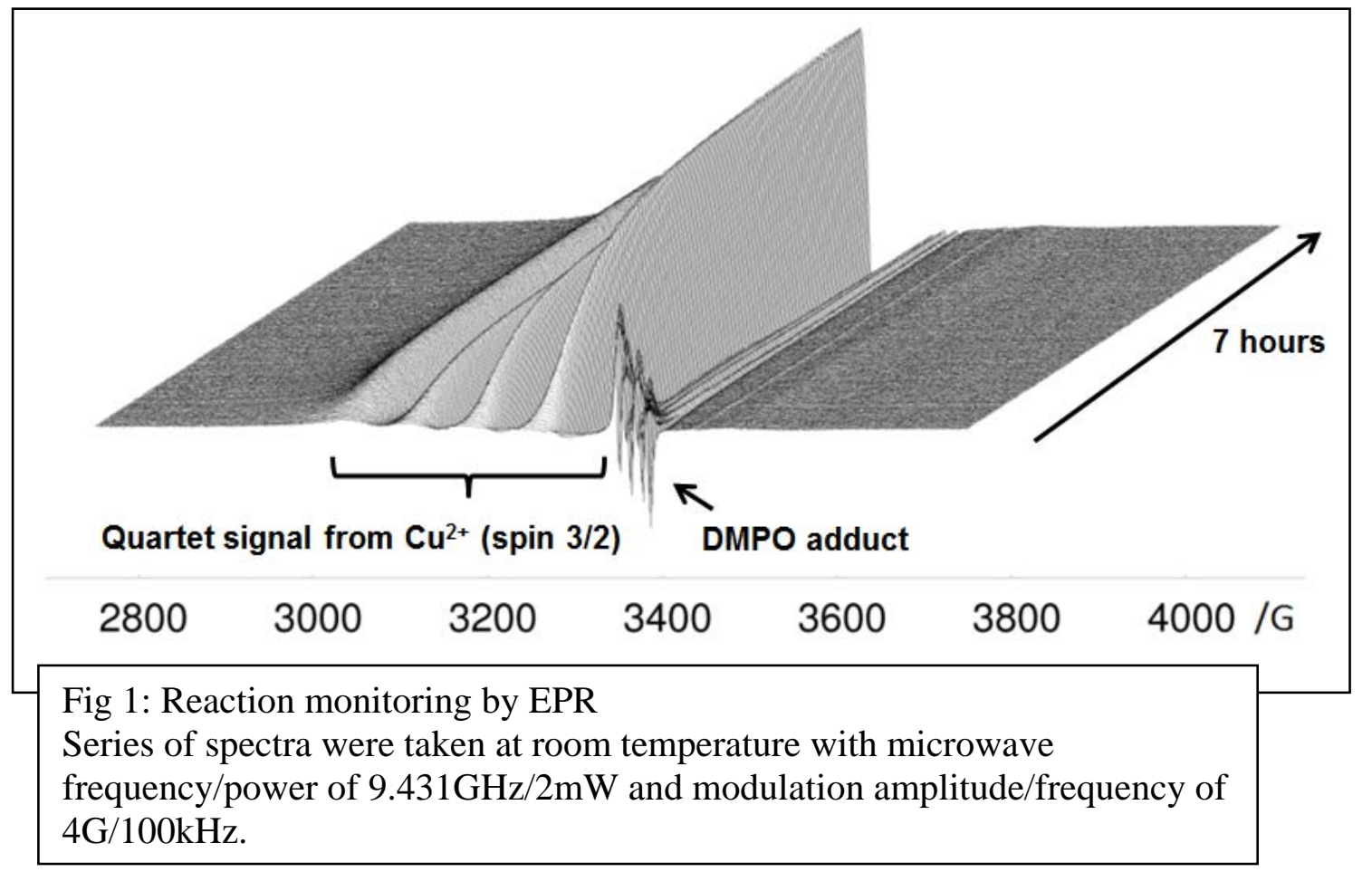

A broad quartet signal between 3000-3300G arises from $\mathrm{Cu}^{2+}$ (spin 3/2) produced during the reaction, which plateaus after $\sim$ 3hours signalling reaction completion. The DMPO adduct produces a sharp multiplet at 3330-3390G, which quickly builds up and then 
decays within an hour. The relatively short signal duration reflects limited stability of the DMPO adduct. Spectral simulation yielded hyperfine coupling constants aN, aH $\beta$, and aH $\gamma$ of 13.3G, 7.7G, and 0.9G respectively.

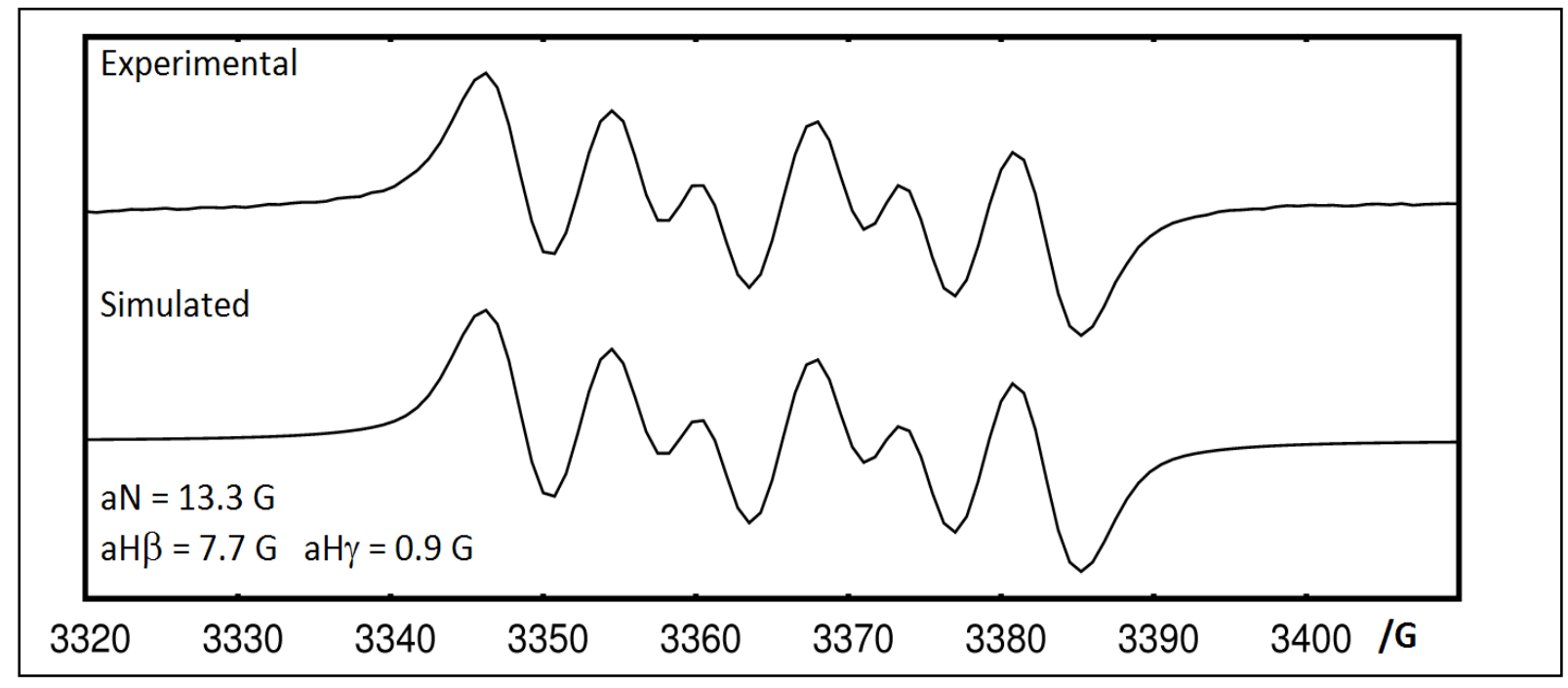

Fig 2: Simulation of the DMPO adduct signal.

At 4 hours, an aliquot of the original mixture was sampled, diluted 100x in MeCN, and analyzed by direct infusion MS. MS (ESI+): calcd for $\mathrm{C}_{10} \mathrm{H}_{20} \mathrm{NO}_{2}[\mathrm{M}+\mathrm{H}] 187$, found 187. 


\section{Mechanisem proposal for $\mathrm{Cu}(\mathrm{OAc}) 2 / \mathrm{TBHP}$ indoline oxidation:}

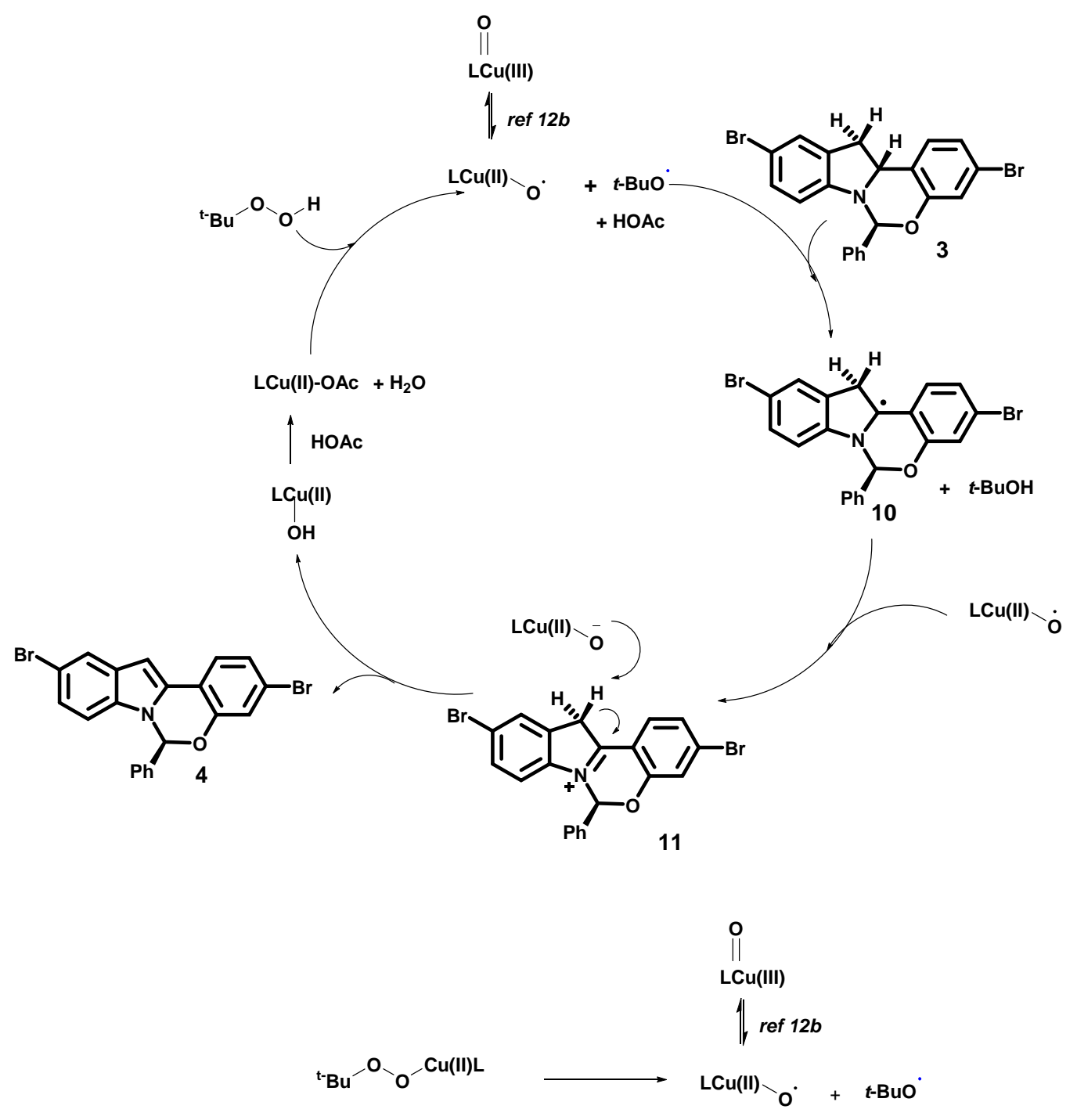




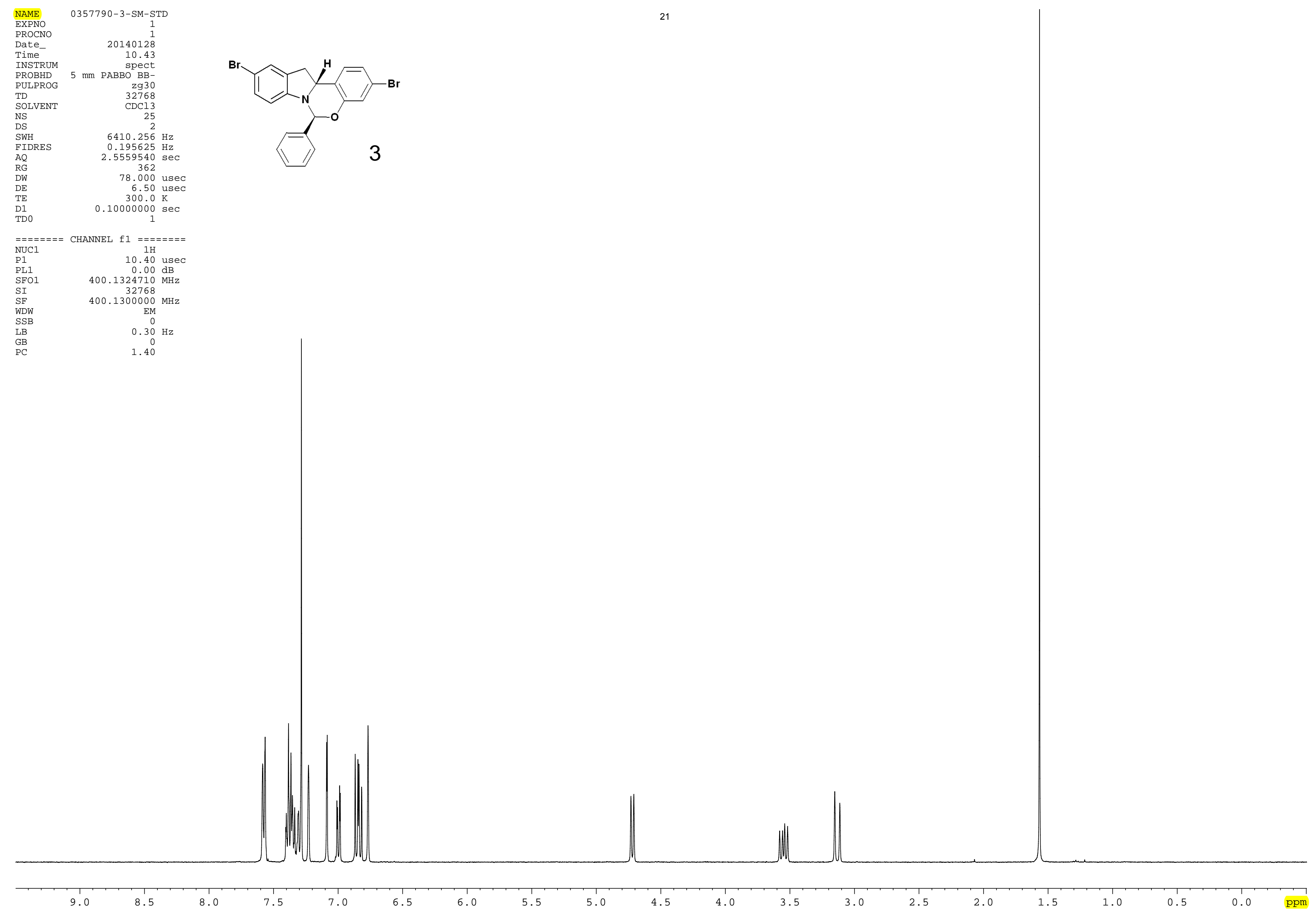




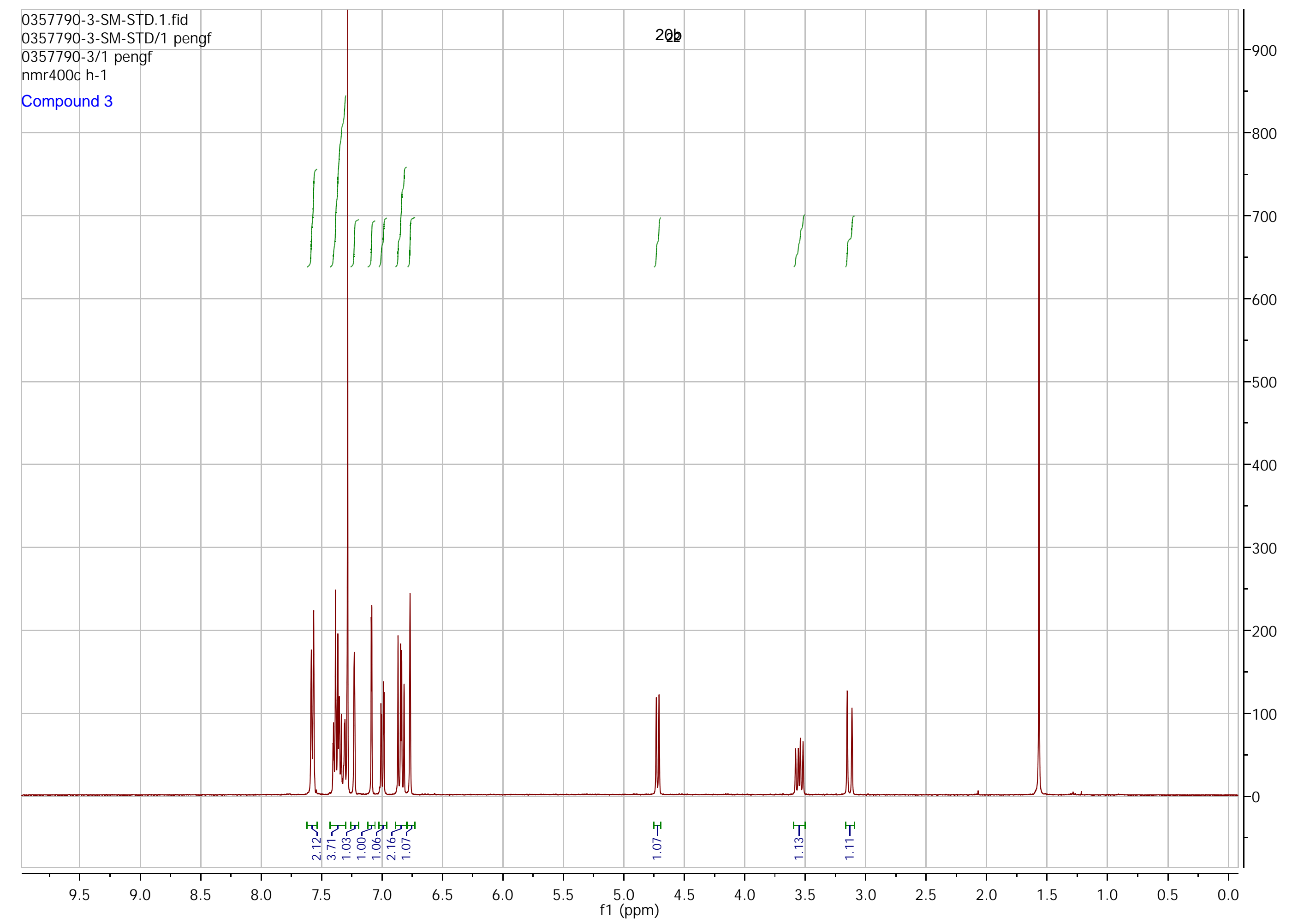


Current Data Parameters

1

Date
Time
INSTRUM
PROBHD

20160209
18.28

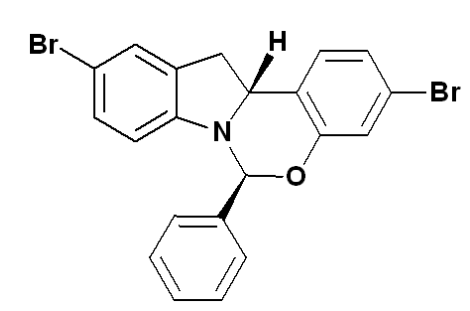

TD

SOLVENT

DS
SWH
FIDRES

$26315.789 \mathrm{~Hz}$ $0.401547 \mathrm{~Hz}$
$1.2452340 \mathrm{sec}$

$\begin{array}{lr}\text { AQ } & 1.2452340 \mathrm{sec} \\ \text { RG } & 2317.5 \\ \text { DW } & 19.600 \mathrm{usec} \\ \text { DE } & 6.50 \mathrm{usec} \\ \text { TE } & 294.6 \mathrm{~K} \\ \text { TE } & 0.109000 \mathrm{sec}\end{array}$

$\begin{array}{lr}\text { AQ } & 1.2432340 \mathrm{sec} \\ \mathrm{RG} & 23170.5 \\ \mathrm{DW} & 19.000 \mathrm{usec} \\ \mathrm{DE} & 6.50 \text { usec } \\ \text { TE } & 294.6 \mathrm{~K}\end{array}$

$\begin{array}{ll}\text { D1 } & 0.10000000 \mathrm{sec} \\ \text { D11 } & 0.03000000 \mathrm{sec}\end{array}$

$\begin{array}{lr}=======\text { CHANNEL } \mathrm{f} 1======== \\ \text { NUC1 } & 13 \mathrm{C} \\ \text { P1 } & 3.80 \mathrm{usec} \\ \text { PL1 } & -6.00 \mathrm{~dB}\end{array}$

$\begin{array}{lr}\text { PL1 } & -6.00 \mathrm{~dB} \\ \text { PL1W } & 127.00000000 \mathrm{~W} \\ \text { SF01 } & 100.6741319 \mathrm{MHZ}\end{array}$

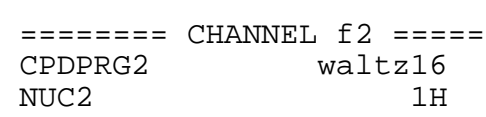

$\begin{array}{lr}\text { NUC2 } & 1 \mathrm{H} \\ \text { PCPD2 } & 80.00 \mathrm{usec} \\ \text { PL2 } & 120.00 \mathrm{~dB}\end{array}$

$\begin{array}{lr}\text { PL2 } & 120.00 \mathrm{~dB} \\ \mathrm{PL12} & 14.12 \mathrm{~dB}\end{array}$

$\begin{array}{lr}\text { PL2W } & 0.00000000 \mathrm{~W} \\ \text { PL12W } & 0.31906110 \mathrm{~W} \\ \text { SF02 } & 400.3320017 \mathrm{MHZ}\end{array}$

$\begin{array}{lr}\text { F2 } & \text { Processing parameters } \\ \text { SI } & 32768 \\ \text { SF } & 100.6630590 \mathrm{MHZ}\end{array}$

$\begin{array}{lc}\text { WF } & 100.6630590 \\ \text { WDW } & \text { no } \\ \text { SSB } & 0 \\ \text { LB } & 0.00 \mathrm{~Hz}\end{array}$

$\begin{array}{ll}\text { LB } & 0.00 \mathrm{~Hz} \\ G B & 0 \\ \text { PC } & 1.40\end{array}$ 


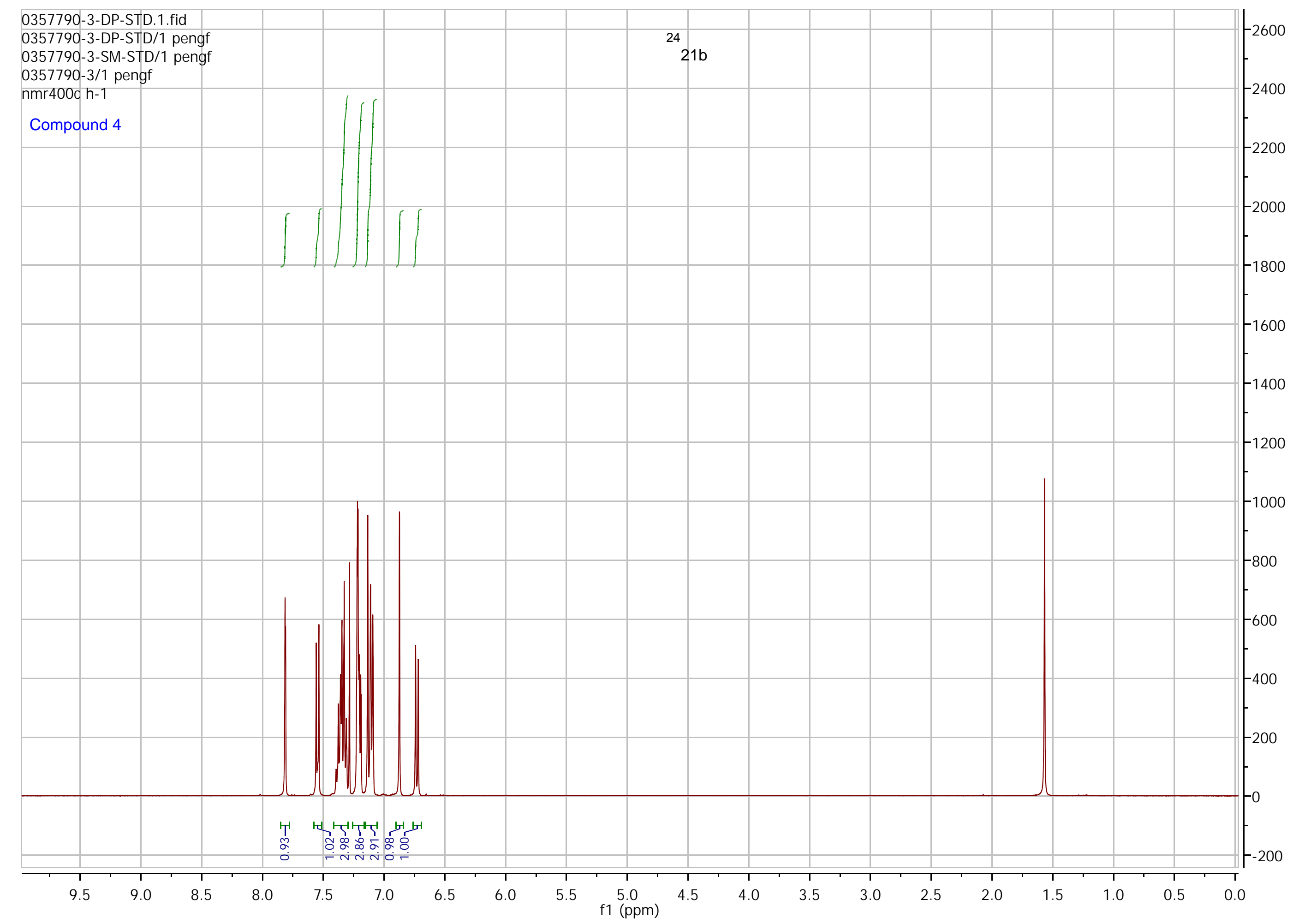




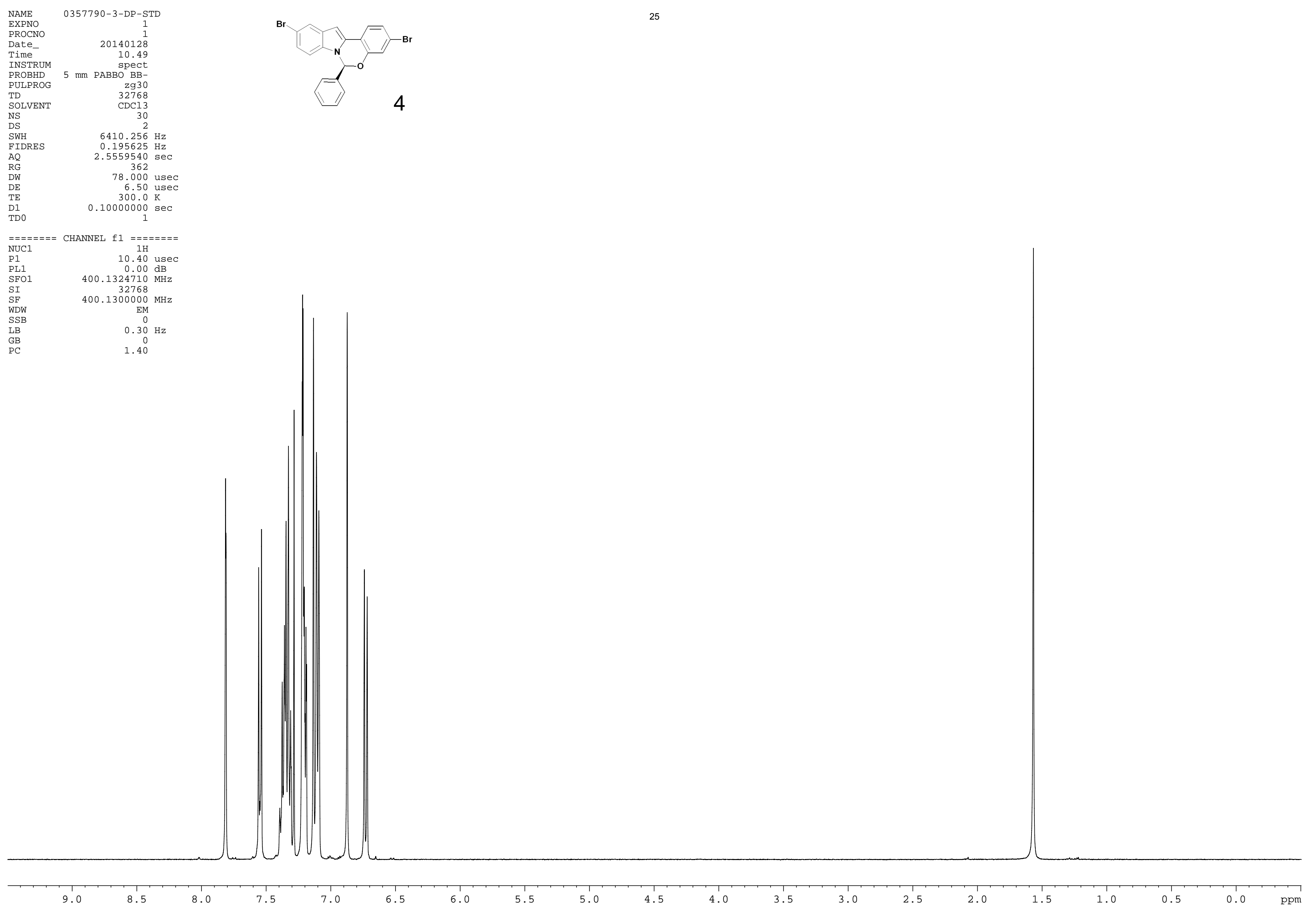


F2 - Acquisition Parameters

10.05
spect

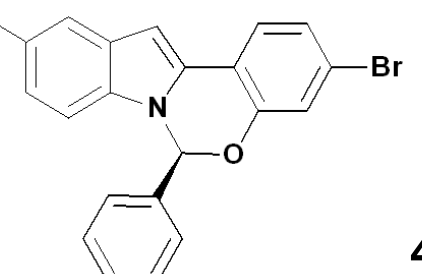

zgdc

SOLVEN

DS
SWH
FIDRES

4

$26315.789 \mathrm{~Hz}$
$0.401547 \mathrm{~Hz}$

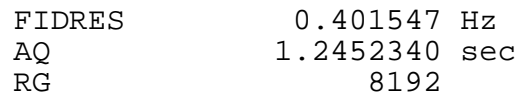

$\begin{array}{lr}\mathrm{DW} & 8192 \mathrm{usc} \\ \mathrm{DE} & 19.000 \mathrm{usec}\end{array}$

TE $\quad 6.50$ us

$\begin{array}{ll}\text { D1 } & 0.10000000 \mathrm{sec} \\ \text { D11 } & 0.03000000 \mathrm{sec}\end{array}$

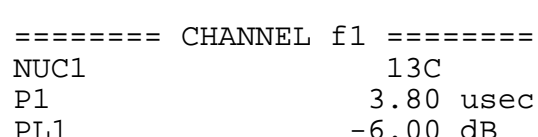

$\begin{array}{lr}\text { PL1 } & -6.00 \mathrm{~dB} \\ \text { PL1W } & 127.00000000 \mathrm{~W} \\ \text { SF01 } & 100.6741319 \mathrm{MHz}\end{array}$

$========$ CHANNEL $\mathrm{f} 2 \mathrm{z}=====$
Waltz16

$\begin{array}{lr}\text { NUC2 } & 1 \mathrm{H} \\ \text { PCPD2 } & 80.00 \mathrm{usec} \\ \text { PL2 } & 120.00 \mathrm{~dB}\end{array}$

$\begin{array}{ll}\text { PL2 } & 120.00 \mathrm{~dB} \\ \text { PL12 } & 14.12 \mathrm{~dB}\end{array}$

$\begin{array}{lr}\text { PL2W } & 0.000000000 \mathrm{~W} \\ \text { PL12W } & 0.31906110 \mathrm{~W} \\ \text { SF02 } & 400.3320017 \mathrm{MHZ}\end{array}$

F2 - Processing parameters

$\begin{array}{ll}\text { SI } & 32768 \\ \text { WF } & 100.6630590 \\ \text { WHW } & \end{array}$

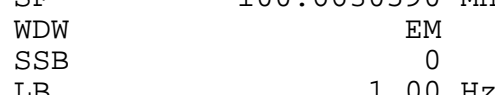

$\begin{array}{lc}\mathrm{LB} & 1.00 \mathrm{~Hz} \\ \mathrm{~GB} & 0 \\ \mathrm{PC} & 1.40\end{array}$ 
Parameter

Title

Spectrometer

Solvent

Temperature

Pulse Sequence

Probe

Number of Scans

Receiver Gain

Relaxation Delay

Pulse Width

Acquisition Time

Acquisition Date

SF01

Spectrometer Frequency 600.13

\section{Spectral Width $\quad 12335.5$}

Lowest Frequency $\quad-2483.0$

Nucleus $\quad 1 \mathrm{H}$

Acquired Size $\quad 32768$

Spectral Size $\quad 65536$

$\mathrm{CDCl} 3$

300.0

32

1.0000

12.0000

6564

\section{Value}

27

5 mm CPPBBO BB-1H/ 19F/ D Z-GRD

Z130038/ 0003

014-03-19T17:02:00 peng 0357790-0051 cdcl3 impurity 1

nmr600 h-1

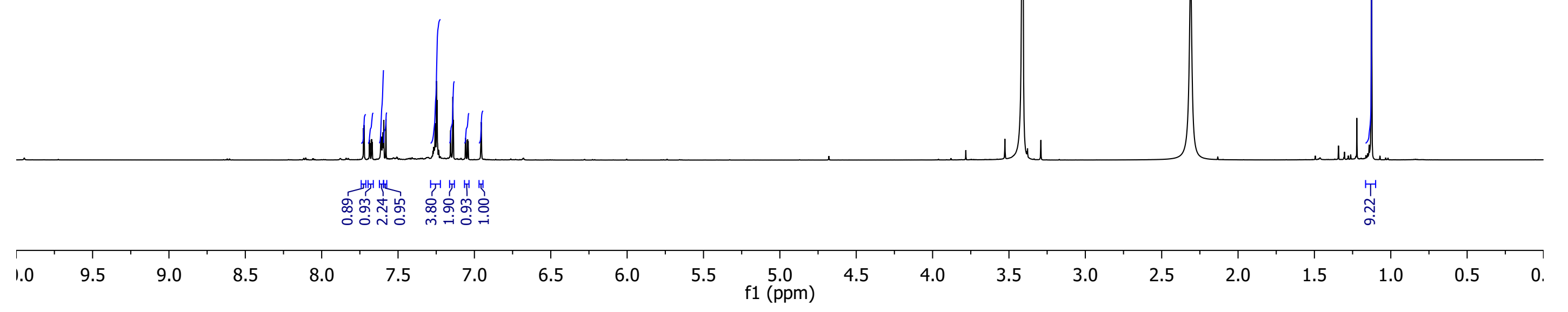


Parameter

Title

\section{Spectrometer}

Solvent

Temperature

Pulse Sequence

Probe

Number of Scans

Receiver Gain

Relaxation Delay

Pulse Width

Acquisition Time

Acquisition Date

SF01

Spectrometer Frequency 150.90

Spectral Width

Lowest Frequency

Nucleus

Acquired Size

Spectral Size

$\mathrm{CDCl} 3$

300.0

zgdc

5 mm CPPBBO BB-1H/ 19F/ D Z-GRD Z130038/ 0003

5120

194

0.1000

4.0000

1.6492

2014-03-20T12:40:00

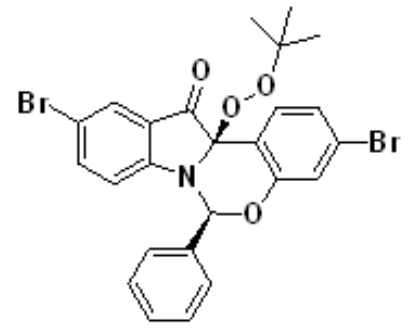

5 peng 0357790-0051 cdcl3

impurity 1

nmr600 c-13 
<smiles>CC1CC1C</smiles> 


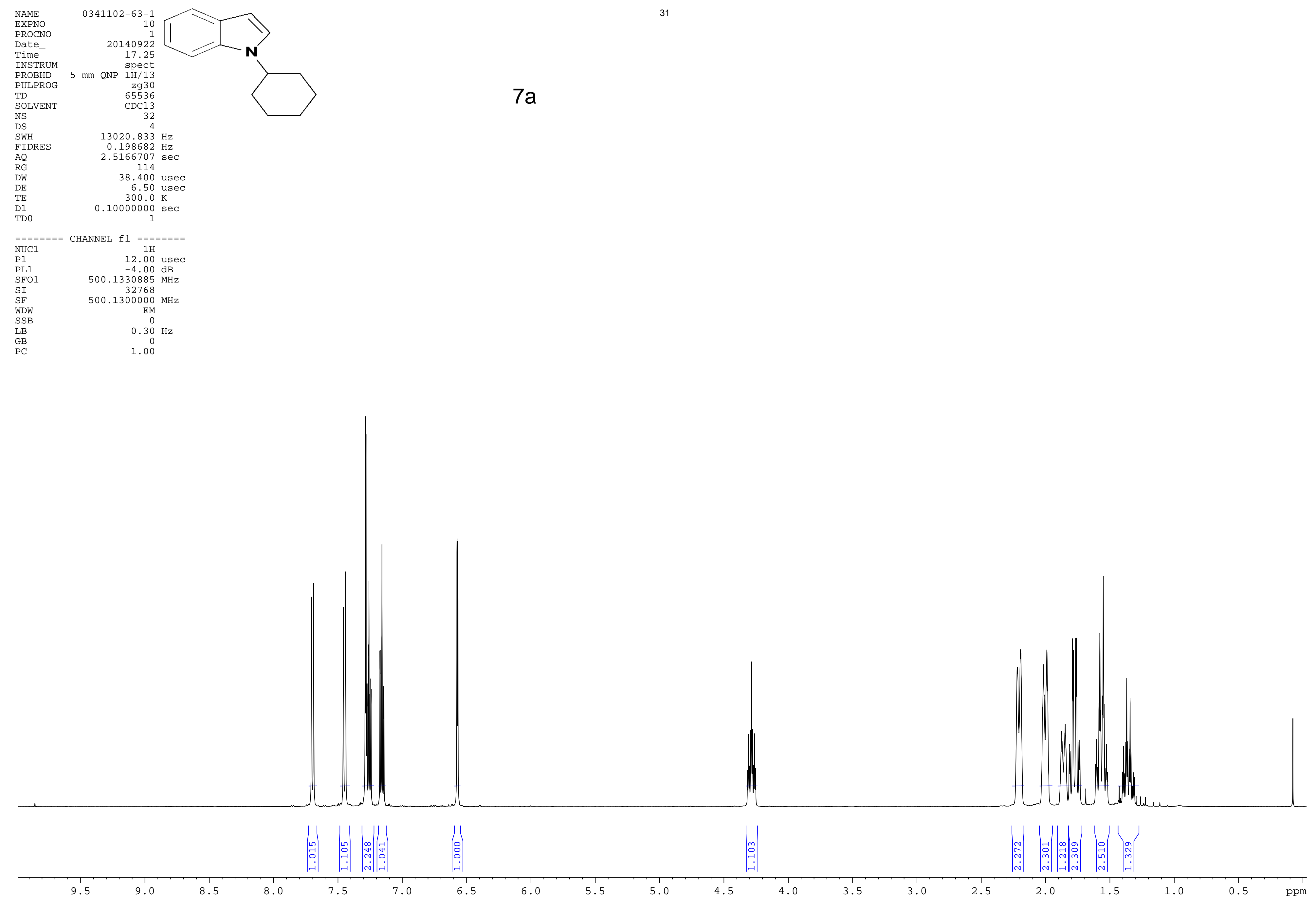




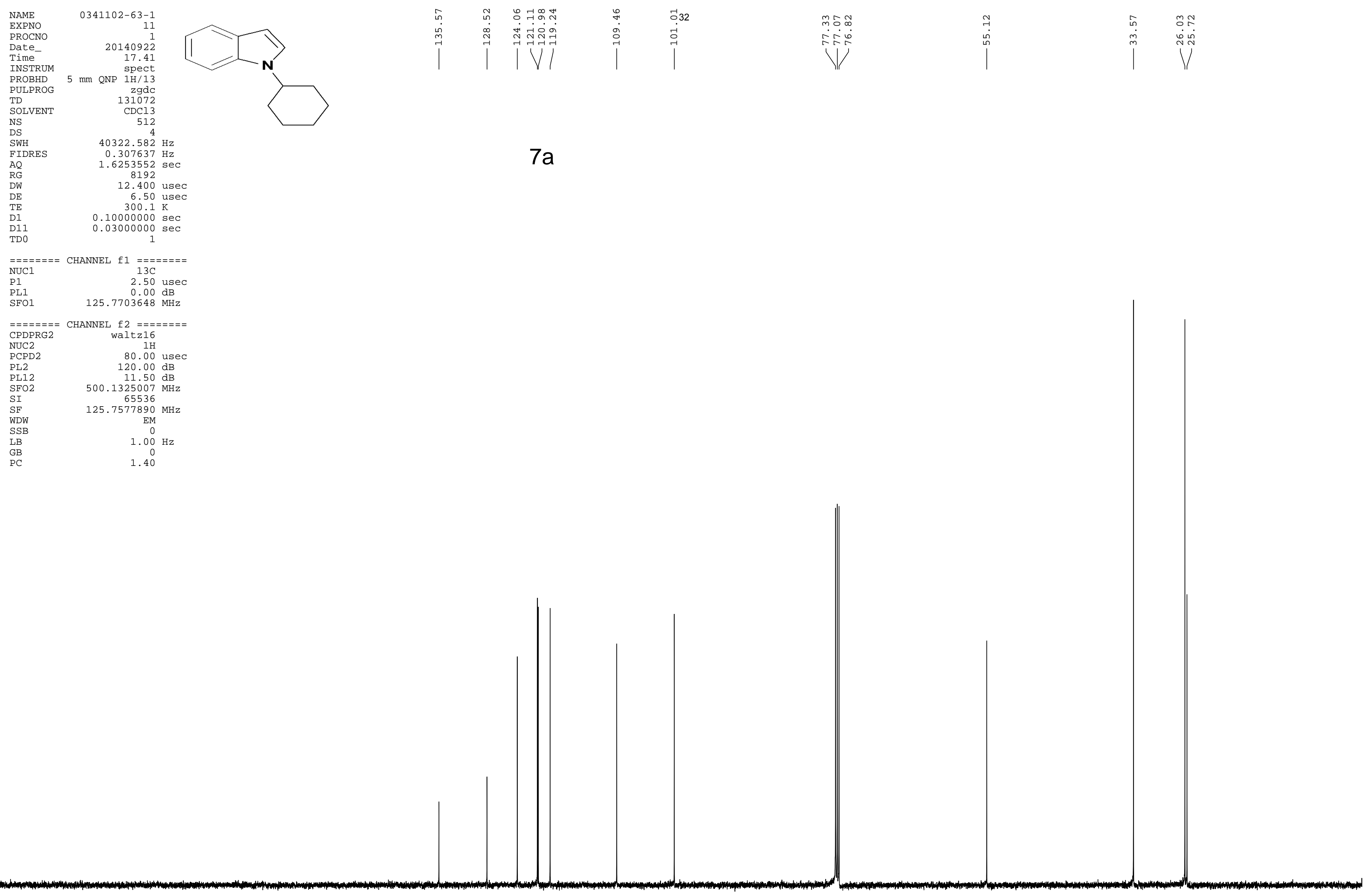


PROCNO

Date

TD

SOLVENT

DS

FIDRES

$\mathrm{RG}$
$\mathrm{DW}$

DE

DD

$=======$ CHANNEL $f 1=======$

SF

WDW
SSB
LB

$\mathrm{PC}$

$6410.256 \mathrm{~Hz}$

$0.195625 \mathrm{HZ}$

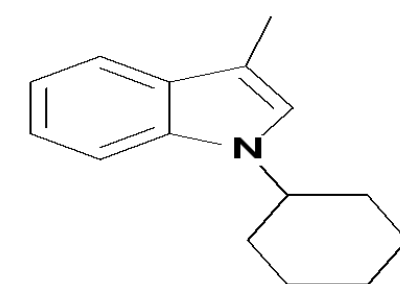

$7 b$

10.40 use
$0.00 \mathrm{~dB}$

$400.1300000 \mathrm{MHZ}$

$\Theta$
$0.30 \mathrm{~Hz}$

0
1.40

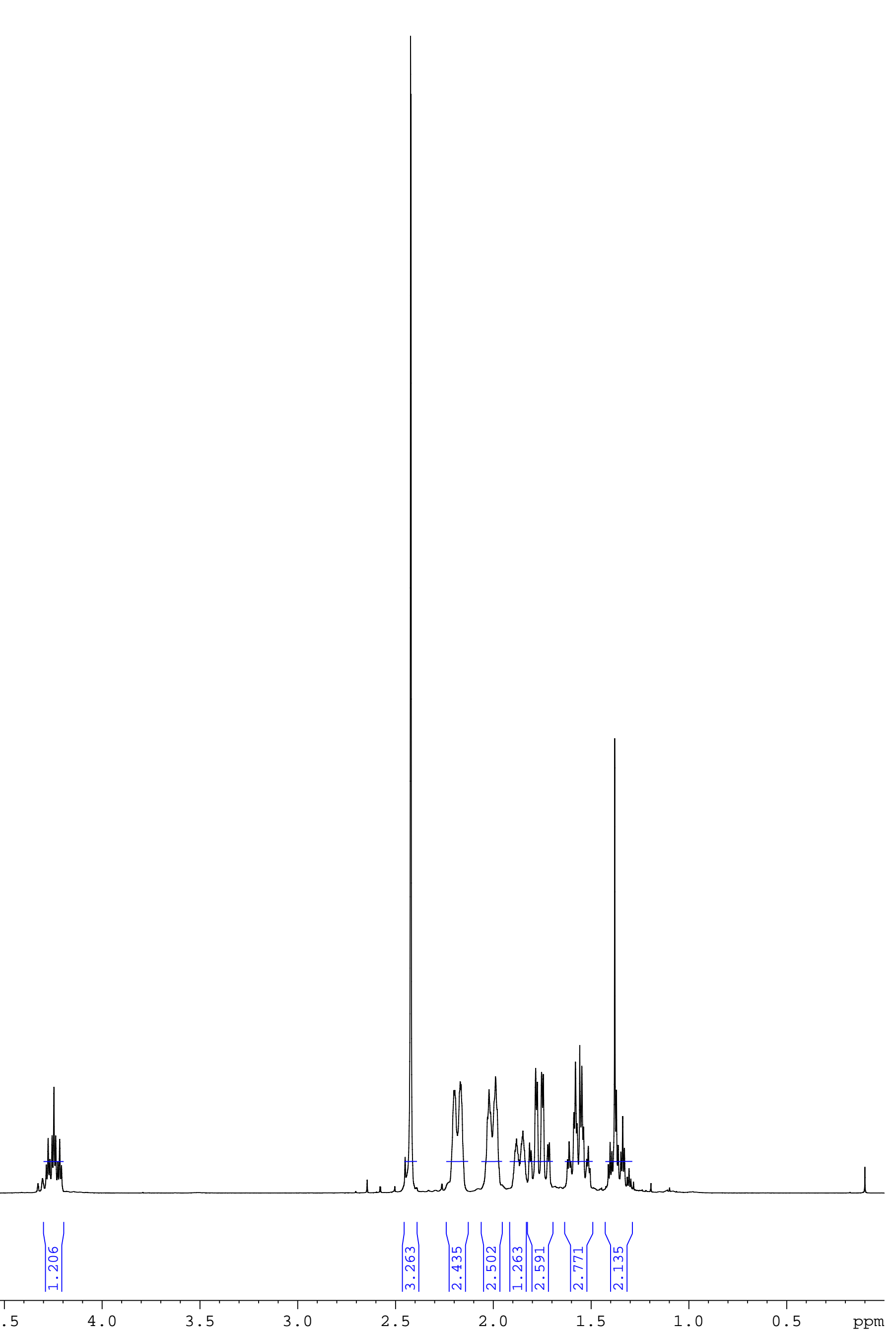




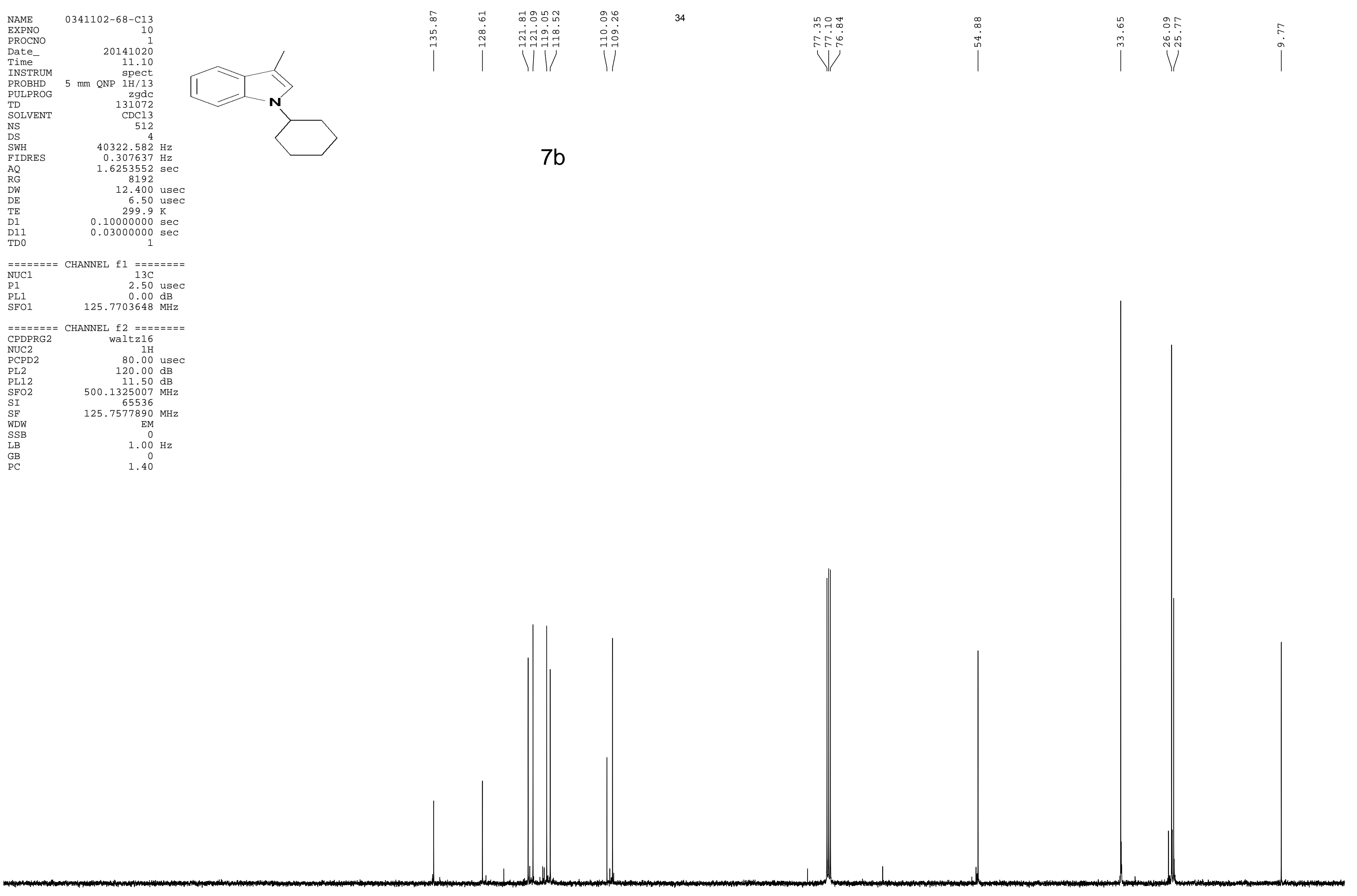



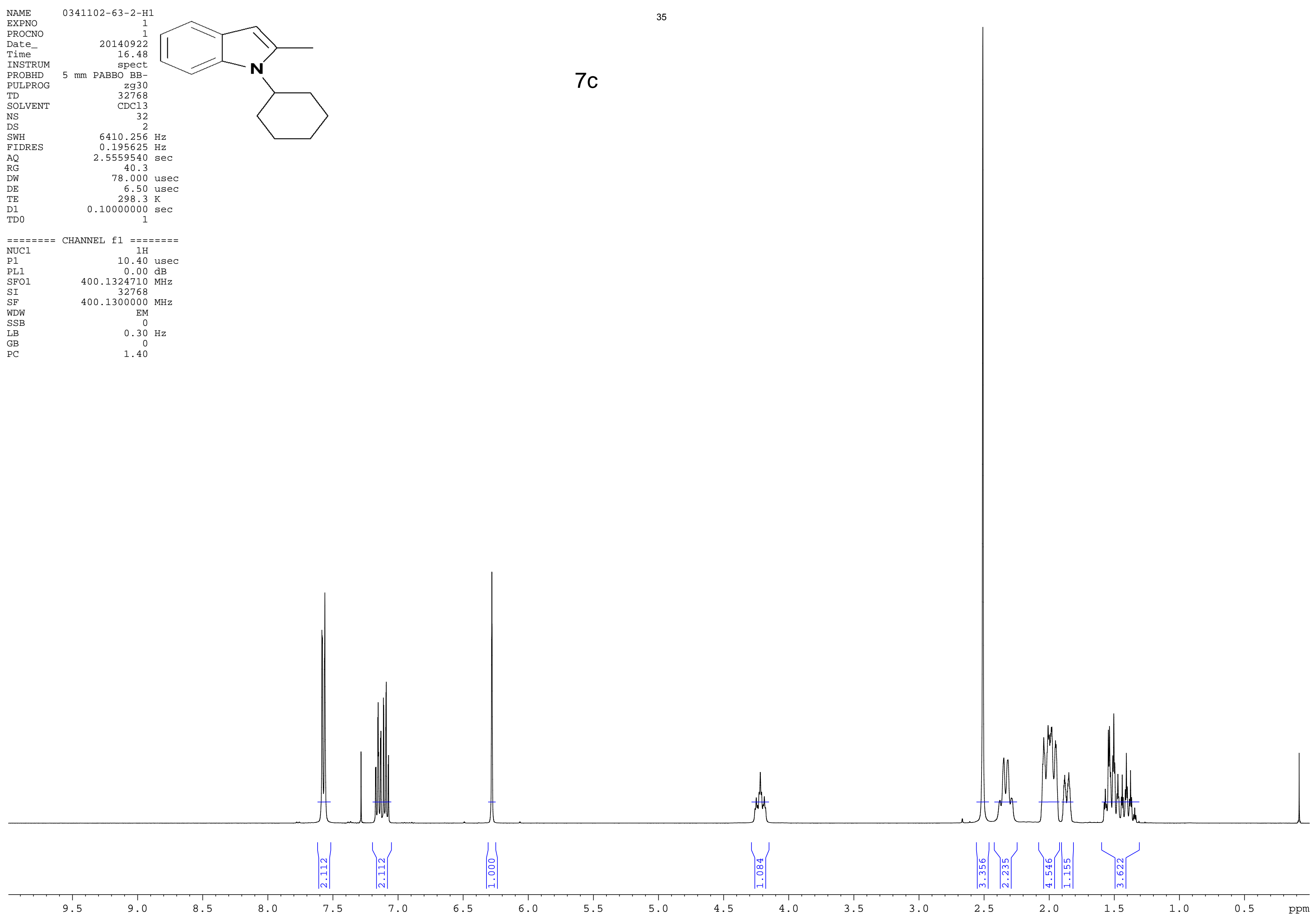


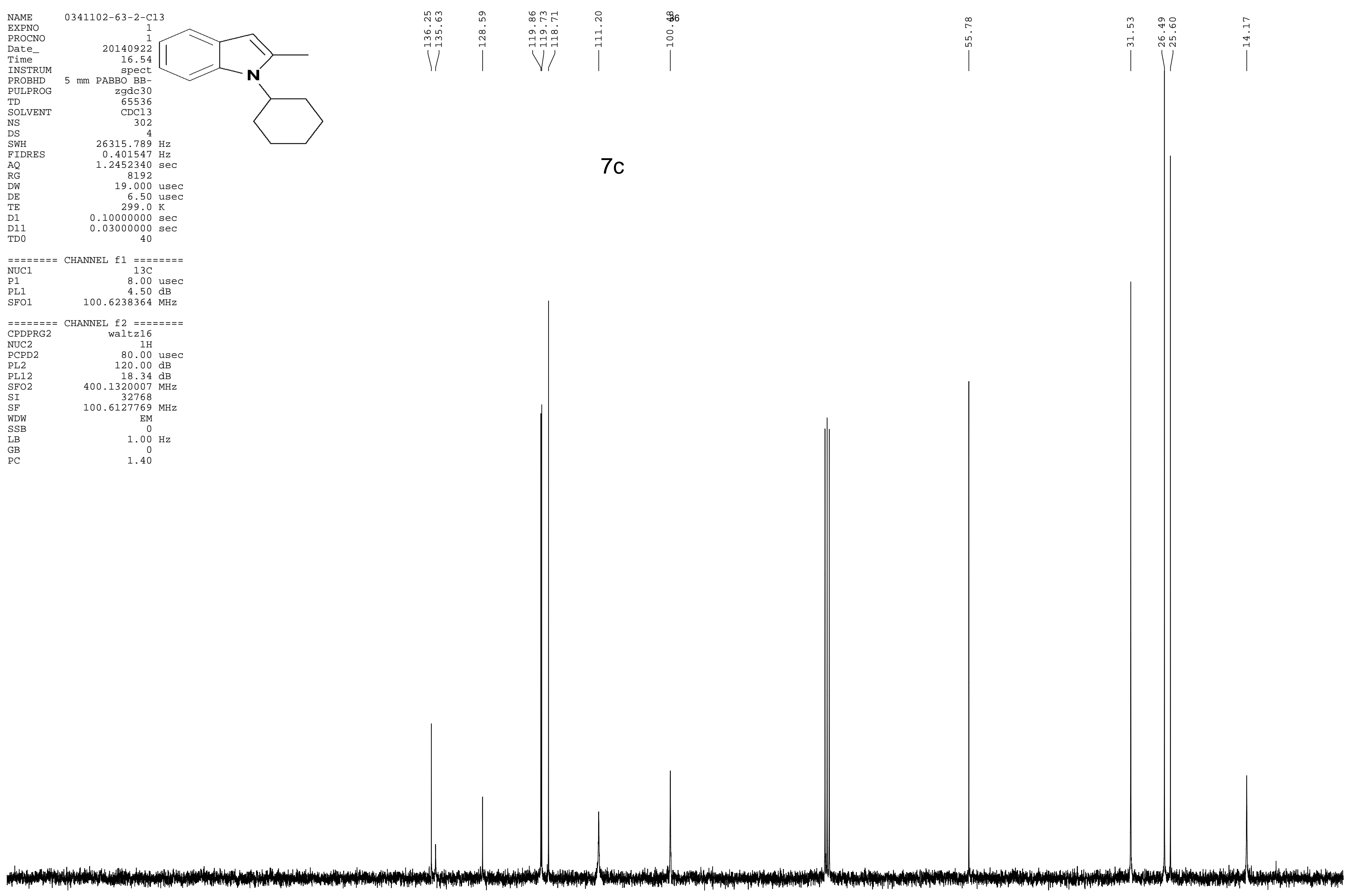




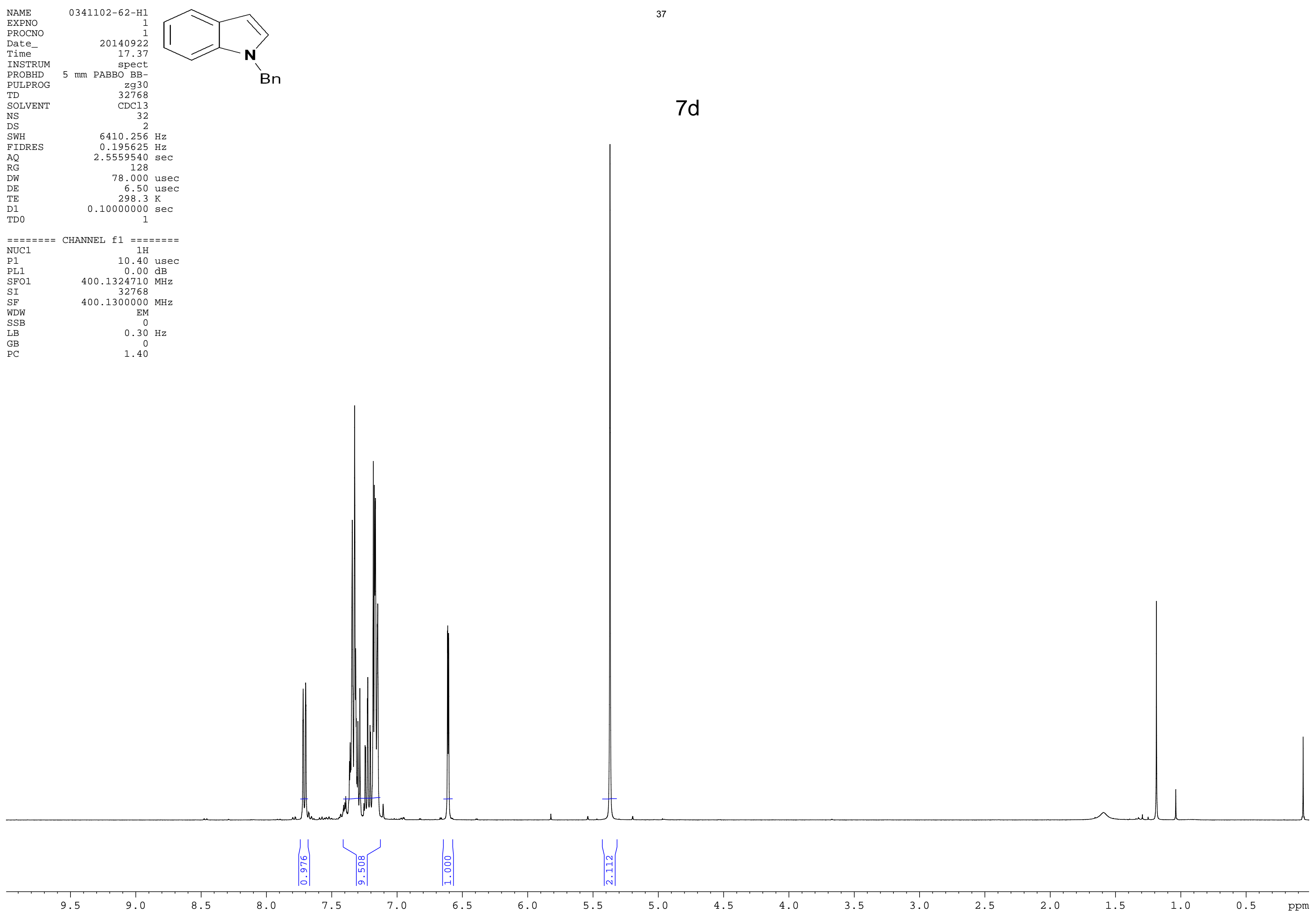




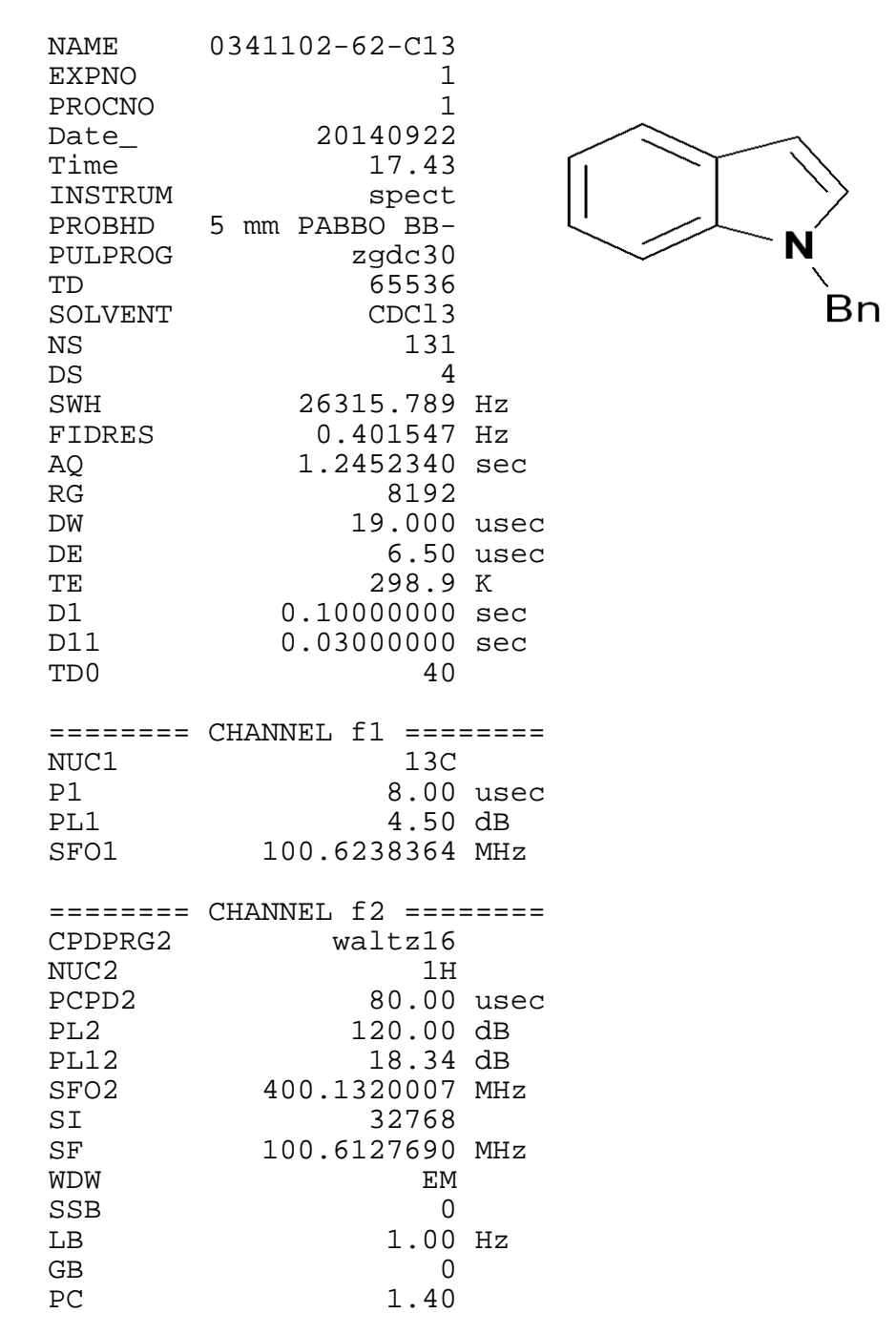




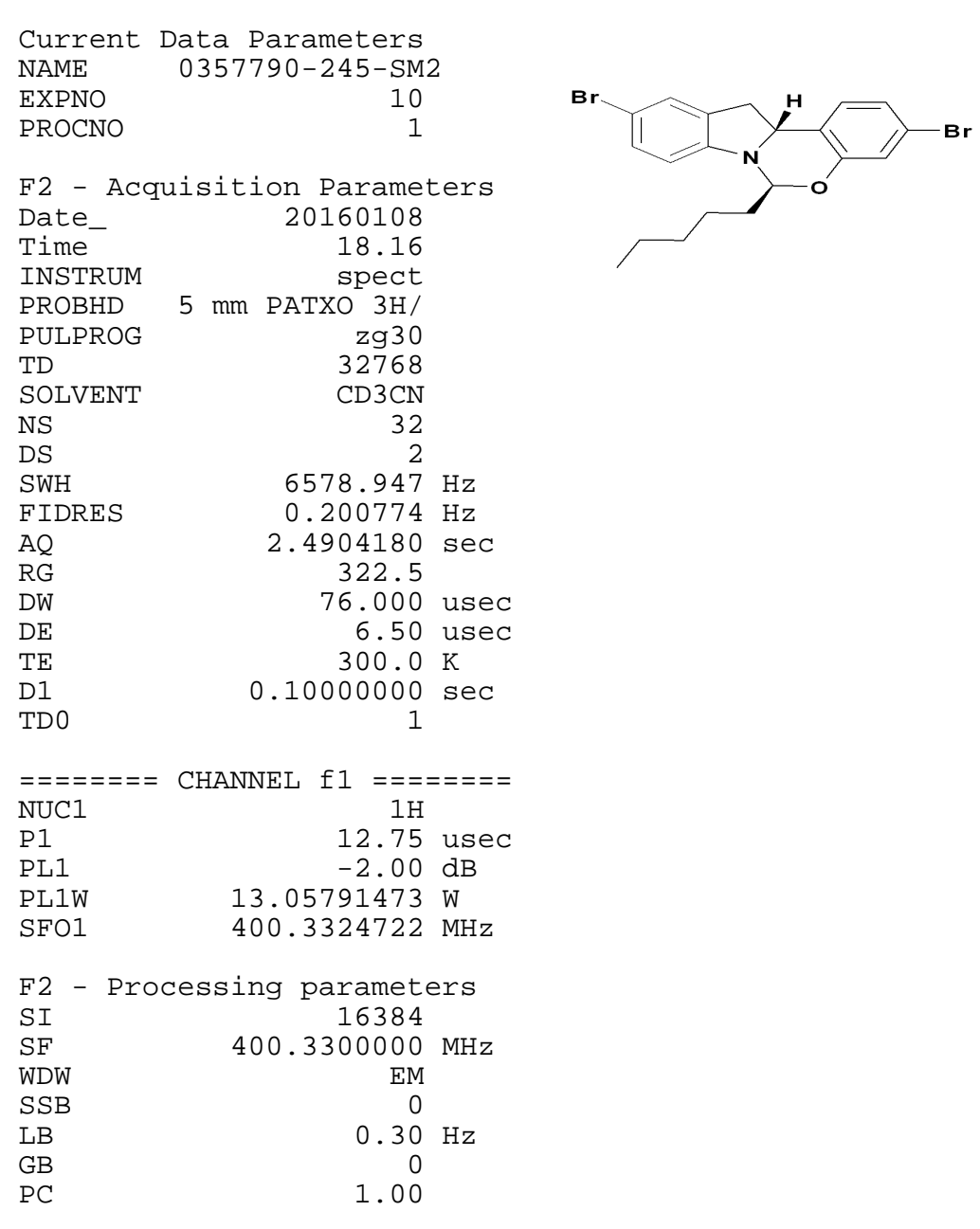

1.00 
Current Data Parameters
$0357790-174$

F2 - Acquisition Parameter

Date

18.45
180202

INSTRU

PROBHD
PULPROG

$\times 03 \mathrm{H} /$

SOLVENT

zgdc
65536

NS

$\begin{array}{ll}\text { FIDRES } & 26315.789 \mathrm{~Hz} \\ \text { AD } & 0.401547 \mathrm{~Hz} \\ \text { A } & 1.2452340 \mathrm{seC}\end{array}$

$\begin{array}{lr}\mathrm{AQ} & 1.2452340 \mathrm{sec} \\ \mathrm{RG} & 18390.4\end{array}$

19.000 usec

$\begin{array}{lr}\text { DE } & 6.50 \mathrm{use} \\ \text { TE } & 294.4 \mathrm{~K} \\ \text { D1 } & 0.10000000 \mathrm{sec} \\ \text { D11 } & 0.03000000 \mathrm{sec}\end{array}$

D11 $\quad 0.03000000$ Sec

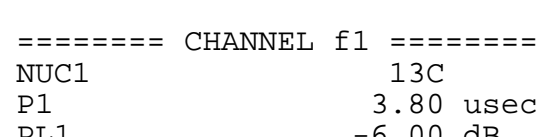

PL1W 127.00000000 W

P $127.00971319 \mathrm{MHz}$

$=======$ CHANNEL $\mathrm{f} 2====$
CPDPRG2
NUC2

$\begin{array}{lr}\text { NUC2 } & \text { Talt } \\ \text { PCPD2 } & 1 \mathrm{H} \\ \text { PL2 } & 80.00 \mathrm{usec} \\ \text { PL12 } & 120.00 \mathrm{~dB} \\ \text { PL2W } & 0.0014 .12 \mathrm{~dB} \\ \text { PL12W } & 0.3190000 \mathrm{~W} \\ \text { SF02 } & 400.3320010 \mathrm{~W} \\ \text { SF2 } & 4 \mathrm{WHz}\end{array}$

F2 - Processing parameter

$\begin{array}{ll}\text { SI } & 32768 \\ \text { SF } & 100.6630599 \\ \text { MHZ }\end{array}$

WDW
SSB
LB

$\mathrm{EM}$

$\mathrm{LB}$
$\mathrm{GB}$
$\mathrm{PC}$

$1 . \stackrel{\ominus}{\mathrm{Hz}}$

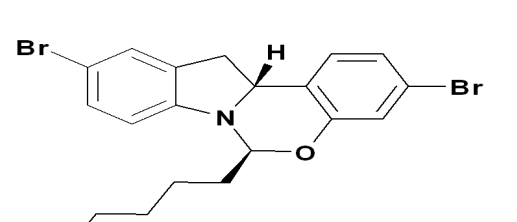

$8 a$

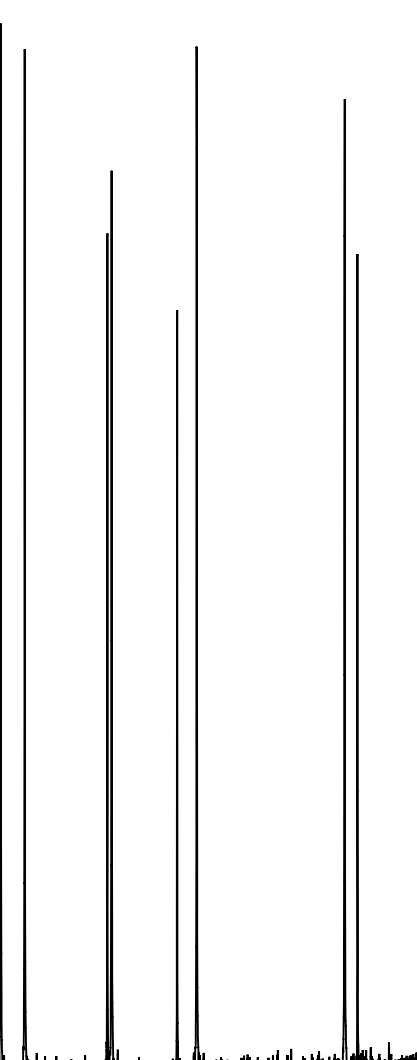




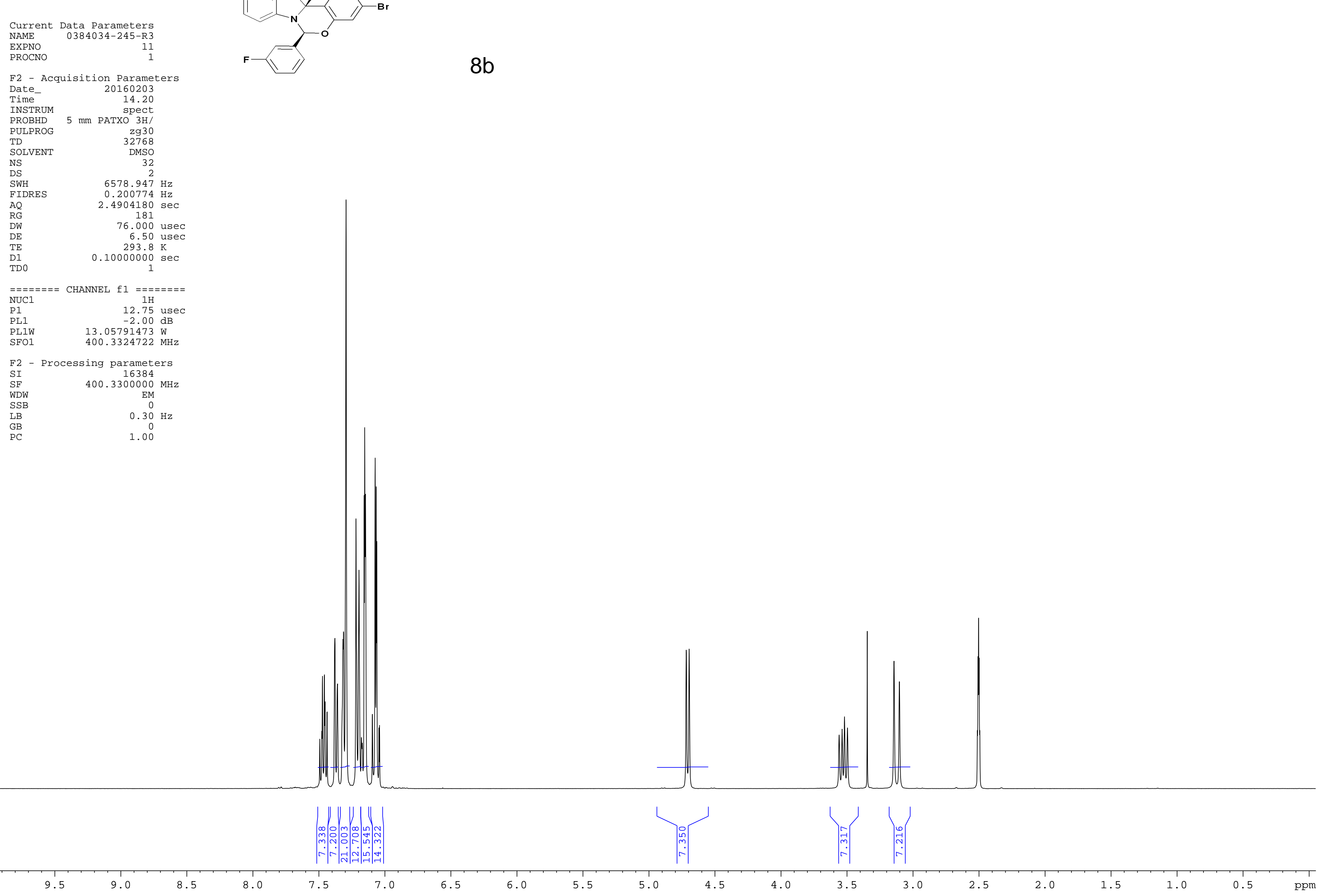


Current Data Parameters
$0357790-176$

$\begin{array}{lr}\text { F2 - } & \text { Acquisition Paramet } \\ \text { Date_- } & 20160205 \\ \text { Time } & 20.06\end{array}$

spect
INSTRUM $5 \mathrm{~mm}$ QNP $1 \mathrm{H} / 13$

PULPROG

zgdc
131072
DMSO
1228

SOLVENT

DS

AQ

$D W$
$D E$
DE
$T E$

TE
D1
D11
TDQ

D11
TD@

$\begin{array}{ll}=======\text { CHANNEL f1 } & 24 \\ \text { NUC1 }====== \\ \text { P1 } & 13 \mathrm{C} \\ \text { P1 } & 2.50 \mathrm{usec}\end{array}$

$\begin{array}{lr}\text { P1 } & 2.50 \text { usec } \\ \text { PL1 } & 0.00 \mathrm{~dB} \\ \text { SF01 } & 125.7703648 \mathrm{MHz}\end{array}$

$=======$ CHANNEL $\mathrm{f} 2=======$
CPDPRG2

$\begin{array}{lc}\text { CPDPRG2 } & \text { waltz16 } \\ \text { NUC2 } & 1 \mathrm{H} \\ \text { PCPD2 } & 80.00 \mathrm{usec} \\ \text { PL2 } & 120.00 \mathrm{~dB} \\ \text { PL12 } & 11.50 \mathrm{~dB}\end{array}$

$\begin{array}{lr}\text { PL12 } & 11.50 \mathrm{~dB} \\ \text { SF02 } & 500.1325007 \mathrm{MHz}\end{array}$

F2 - Processing parameters

$\begin{array}{lr}\text { SI } & 65536 \\ \text { SF } & 125.7577890 \mathrm{MHz}\end{array}$

$\begin{array}{lc}\text { WDW } & 125.757789 \\ \text { SSB } & \text { EM } \\ \text { LB } & 1.00 \mathrm{~Hz} \\ \text { CB } & \end{array}$

$\begin{array}{cc}\mathrm{LB} & 1.00 \mathrm{~Hz} \\ \mathrm{~GB} & 0 \\ \mathrm{PC} & 1.40\end{array}$

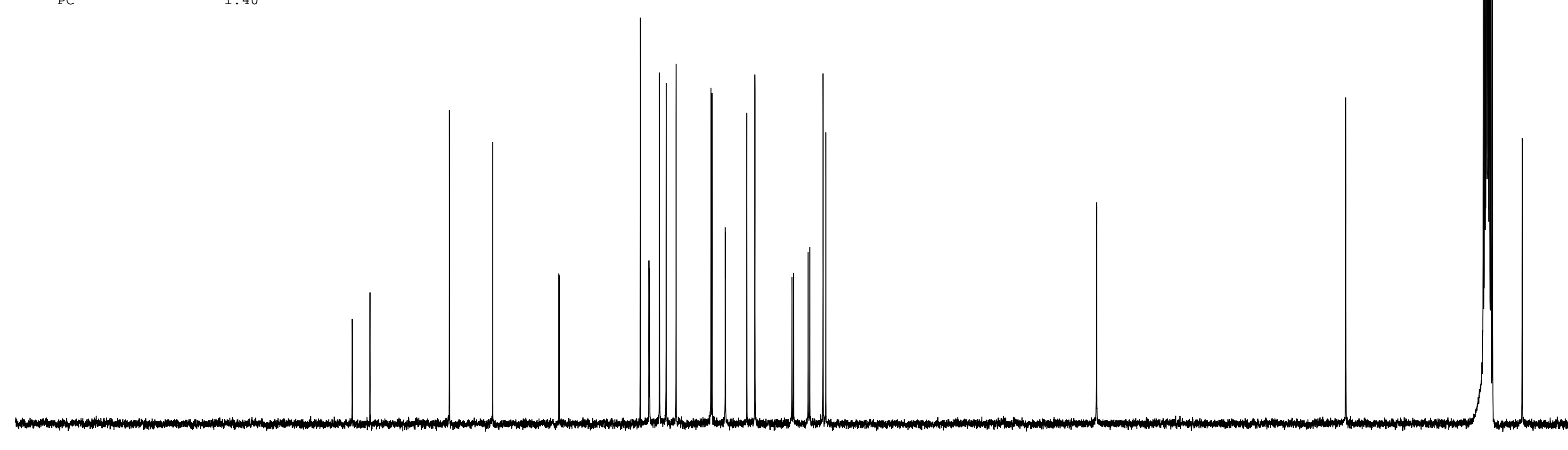




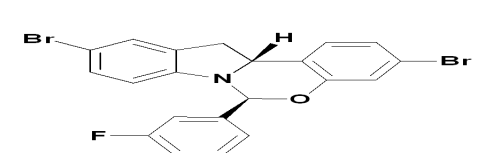

Current Data Parameters
NAME

EXPNO
PROCNO

F2 - Acquisition Parameters

Time 20160205
5.37

$5 \mathrm{~mm}$ QNP $1 \mathrm{H} / 13$

PULPROG

$1 \mathrm{~h} / 13$

SOLVEN

$\begin{array}{lr}\text { DS } & 4 \\ \text { SWH } & 141843.969 \mathrm{~Hz}\end{array}$

$1.082184 \mathrm{~Hz}$
$0.4620788 \mathrm{seC}$

RQ 64
3.525
5.04
3.04

$300.1 \mathrm{~K}$

$\begin{array}{ll}\text { TE } & 0.10000000 \mathrm{sec} \\ \text { D1 } & 0.03000000 \mathrm{sec} \\ \text { D11 } & \end{array}$

D12 $\quad 0.00002000 \mathrm{sec}$

$=======$ CHANNEL $f 1=======$
NUC1 $19 \mathrm{~F}$

$\begin{array}{lr}\text { NUC1 } & 19 \mathrm{~F} \\ \text { P1 } & 5.00 \mathrm{usec} \\ \text { PL1 } & -4.00 \mathrm{~dB}\end{array}$

SF01 470.5217881 MHZ

$=======$ CHANNEL $\mathrm{f} 2=======$
waltz16

$\begin{array}{ll}\text { NUC2 } & 1 \mathrm{H} \\ \text { PCPD2 } & 85.00 \mathrm{usec}\end{array}$

$\begin{array}{lr}\text { PCPD2 } & 85.00 \mathrm{usec} \\ \text { PL2 } & 120.00 \mathrm{~dB} \\ \text { PL12 } & 12.00 \mathrm{~dB} \\ \text { SH12 } & \end{array}$

$\begin{aligned} 12.00 \mathrm{~dB} & \\ 0.1325007 \mathrm{MHz} & \end{aligned}$

F2 - Processing parameters

$\begin{array}{lr}\text { SI } & 65536 \\ \mathrm{SF} & 470.5923770 \mathrm{MHZ}\end{array}$

$\begin{array}{lc}\text { SF } & 470.5923770 \mathrm{MHz} \\ \text { WDW } & \mathrm{EM} \\ \text { SSB } & 0 \\ \text { LB } & 1.00 \mathrm{~Hz} \\ \text { CB } & 1.00\end{array}$

$\begin{array}{lc}1.00 \\ \mathrm{~GB} & 0 \\ \mathrm{PC} & 1.00\end{array}$ 


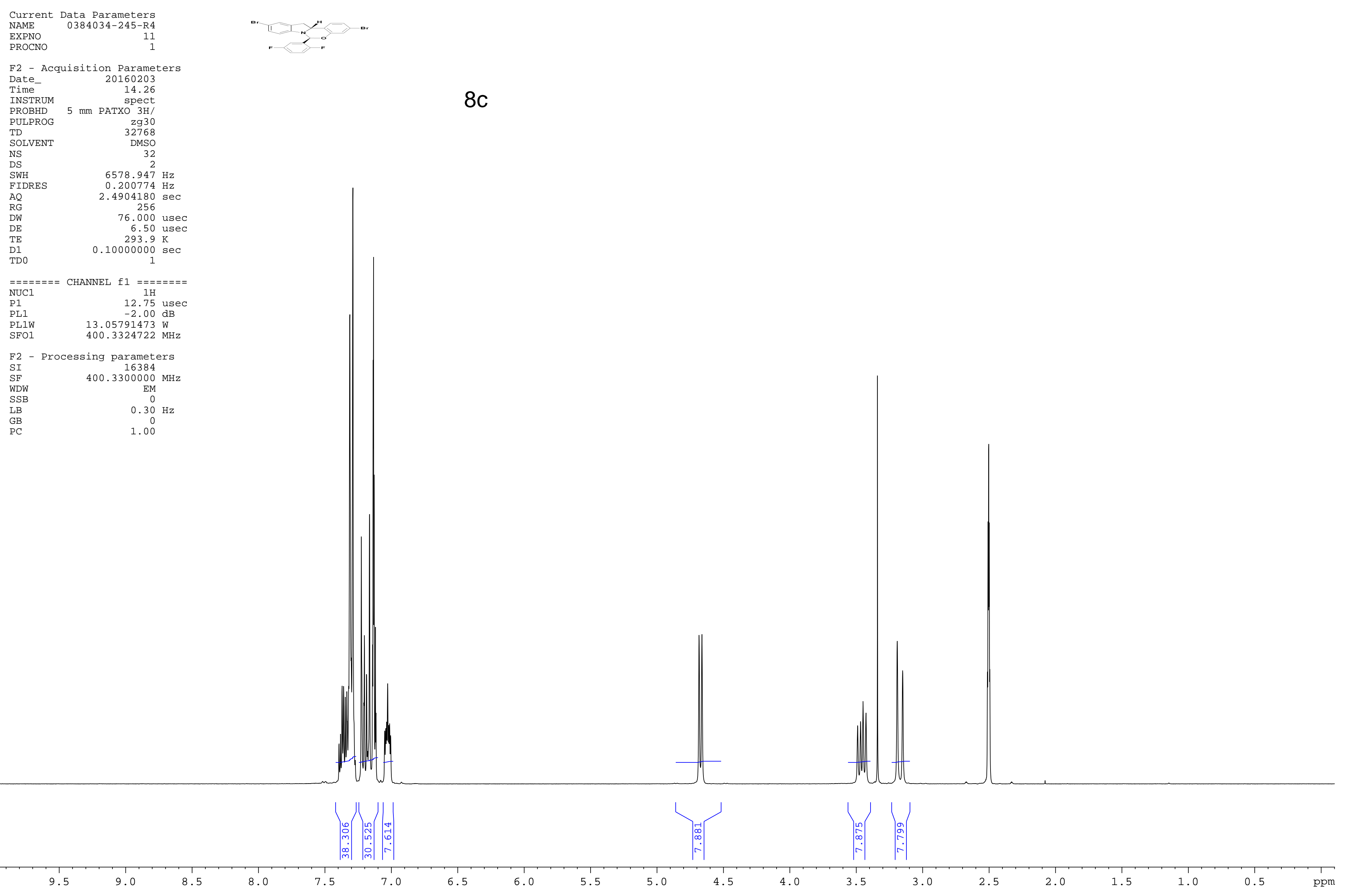


Current Data Parameters
NAME

ameters

Date ${ }^{-}$

$\begin{array}{lr}20160206 \\ \text { INSTRUM } & 2.15\end{array}$

PROBHD

mm QNP $1 \mathrm{Hect}$

$8 c$

TD

131072
DMSO
12288

NS

$\begin{array}{ll}\text { DS } & 4 \\ \text { SWH } & 40322.582 \mathrm{~Hz}\end{array}$

$\begin{array}{lr}\text { AQ } & 0.307637 \mathrm{~Hz} \\ \text { RG } & 1.6253428 \mathrm{sec}\end{array}$

$\begin{array}{lr}\mathrm{RG} & 8192 \\ \mathrm{DW} & 12.400 \mathrm{usec}\end{array}$

DE

$\begin{array}{ll}\text { D1 } & 0.10000000 \mathrm{sec} \\ \text { D11 } & 0.03000000 \mathrm{sec}\end{array}$

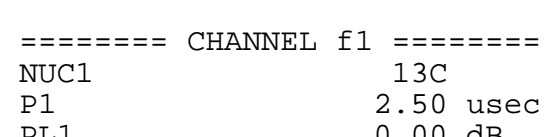

$\begin{array}{lr}\text { P1 } & 2.50 \text { usec } \\ \text { PL1 } & 0.00 \mathrm{~dB} \\ \text { SF01 } & 125.7703648 \mathrm{MHz}\end{array}$

$========$ CHANNEL $\mathrm{f} 2========$
waltz16

$\begin{array}{lc}\text { CPDCRG2 } & \text { Waltz16 } \\ \text { NUC2 } & 1 \mathrm{H} \\ \text { PCPD2 } & 80.00 \mathrm{usec} \\ \text { PL2 } & 120.00 \mathrm{~dB} \\ \text { PL12 } & 11.50 \mathrm{~dB}\end{array}$

$\begin{array}{lr}\text { PL2 } & 120.50 \mathrm{~dB} \\ \text { PL12 } & 11.500 .1325007 \mathrm{MHz}\end{array}$

F2 - Processing parameters

$\begin{array}{lr}\text { SI } & 65536 \\ \text { SF } & 125.7577890 \mathrm{MHz}\end{array}$

$\begin{array}{lc}\text { WDW } & \text { EM } \\ \text { SSB } & 0 \\ \text { LB } & 1.00 \mathrm{~Hz} \\ \text { CB } & 1.40\end{array}$

$\begin{array}{lr}\text { GB } & 1.00 \\ \text { GB } & 0 \\ \text { PC } & 1.40\end{array}$ 

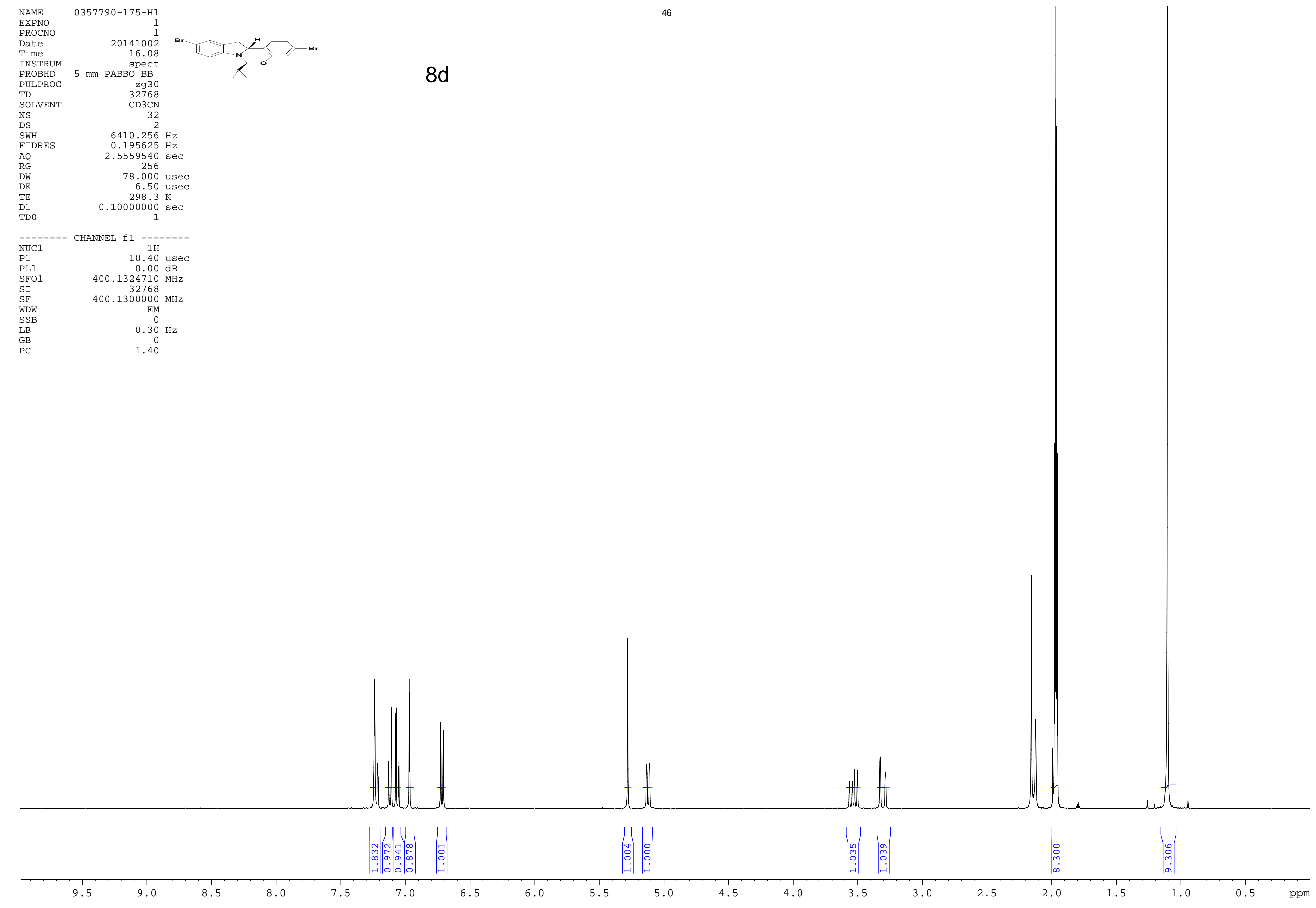
20160203
10.27

Time-
INSTRUM

spect
INSTRUM 5 mm PATXO 3 H

PROBHD
PULPROG

SOLVENT

NS

DWH
FIDRES

$\begin{array}{lr}\text { AQ } & 0.401547 \mathrm{~Hz} \\ \mathrm{RG} & 1.2452340 \mathrm{sec}\end{array}$

$\begin{array}{lr}R G & 8192 \\ D W & 19.000 \text { usec } \\ \text { DE } & 6.50 \text { usec }\end{array}$

$294.4 \mathrm{~K}$

$\begin{array}{ll}0.10000000 \mathrm{sec} \\ \text { D11 } & 0.03000000 \mathrm{sec} \\ \text { TDO } & \end{array}$

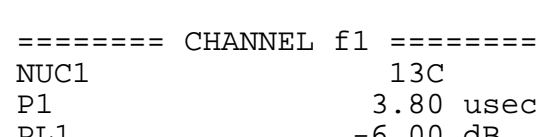

PL1
PL1W 127. $00000000 \mathrm{~dB}$
SF01

100.6741319 MHz

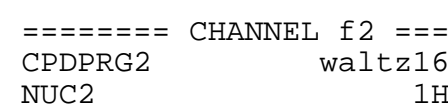

$\begin{array}{lr}\text { NUC2 } & \text { TH } \\ \text { PCPD2 } & 80.00 \mathrm{usec} \\ \text { PL2 } & 120.00 \mathrm{~dB} \\ \text { PL12 } & 14.12 \mathrm{~dB} \\ \text { PL2W } & 0.0000000 \mathrm{~W} \\ \text { PL12W } & 0.31906110 \mathrm{~W} \\ \text { PF02 } & 400.3320017 \mathrm{MHz}\end{array}$

F2 - Processing parameters

$\begin{array}{lr}\text { SI } & 32768 \\ \text { SF } & 100.6630590 \mathrm{MHz} \\ \text { WDW } & \mathrm{EM}\end{array}$

$\begin{array}{lc}\text { SSB } & 0 \\ \text { LB } & 1.00 \mathrm{~Hz} \\ \text { GB } & 0\end{array}$

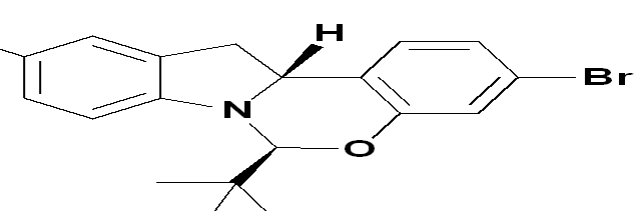

$8 d$
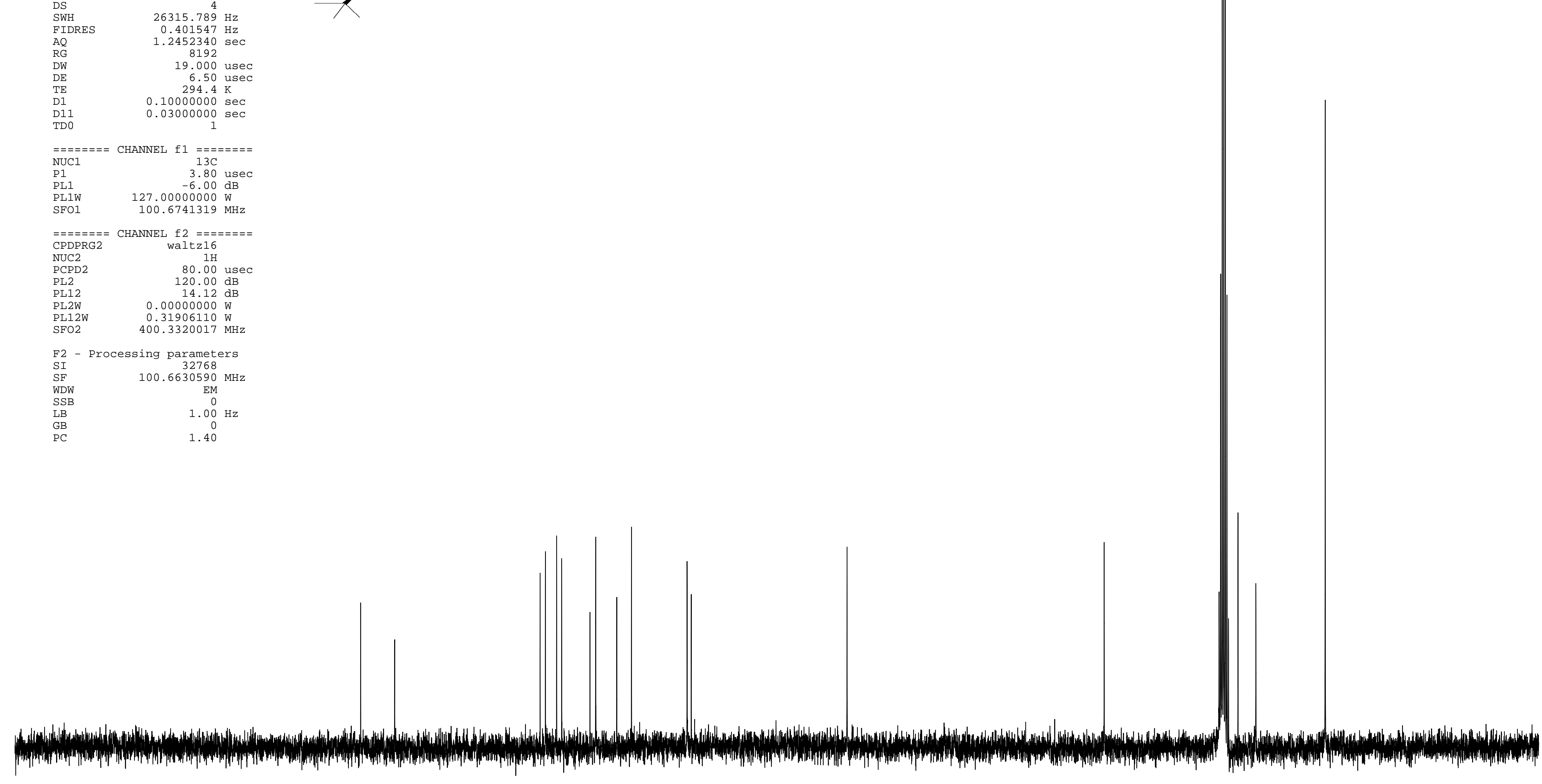
$\begin{array}{cc}\text { F2 - Acquisition Parameters } \\ \text { Date } \\ \text { Time } & 20160205 \\ \text { Tims } & 5.33\end{array}$

spect
$1 \mathrm{~m} / 13$

PULPROG
TD

fhiggn
131072

SOLVENT

$\begin{array}{lr}\text { DS } & 4 \\ \text { SWH } & 141843.969 \mathrm{HZ} \\ \text { FIDRES } & 1.4621848 \mathrm{~Hz}\end{array}$

$1.082184 \mathrm{~Hz}$
$0.4620788 \mathrm{sec}$

RQ

3.525 usec

$300.1 \mathrm{~K}$

$\begin{array}{ll}\text { D1 } & 0.10000000 \mathrm{sec} \\ \text { D11 } & 0.03000000 \mathrm{sec}\end{array}$

$00002000 \mathrm{sec}$

$=======$ CHANNEL $f 11=======$
NUC1 $19 \mathrm{~F}$

$\begin{array}{lr}\text { P1 } & 5.00 \text { usec } \\ \text { PL1 } & -4.00 \mathrm{~dB}\end{array}$

$0.5217881 \mathrm{MHz}$

$=======$ CHANNEL $\mathrm{f} 2 \mathrm{z}======$
waltz16

NUC2
PCPD2

$\begin{array}{lr}\text { PL2 } & 85.00 \text { usec } \\ \text { PL12 } & 120.00 \mathrm{~dB}\end{array}$

SF02 $500.1325007 \mathrm{MHZ}$

F2 - Processing parameters

$\begin{array}{lr}\text { SI } & 65536 \\ \mathrm{SF} & 470.5923770 \mathrm{MHz}\end{array}$

$\begin{array}{lr}\text { SF } & 470.5923770 \mathrm{MHZ} \\ \text { WDW } & \mathrm{EM} \\ \text { SSB } & 0\end{array}$

$\begin{array}{lc}\text { LSB } & 0 \\ \text { LB } & 1.00 \mathrm{HZ} \\ \mathrm{PC} & 0\end{array}$

$$
\begin{aligned}
& 1.00 \mathrm{~Hz} \\
& 0 \\
& 1.00
\end{aligned}
$$

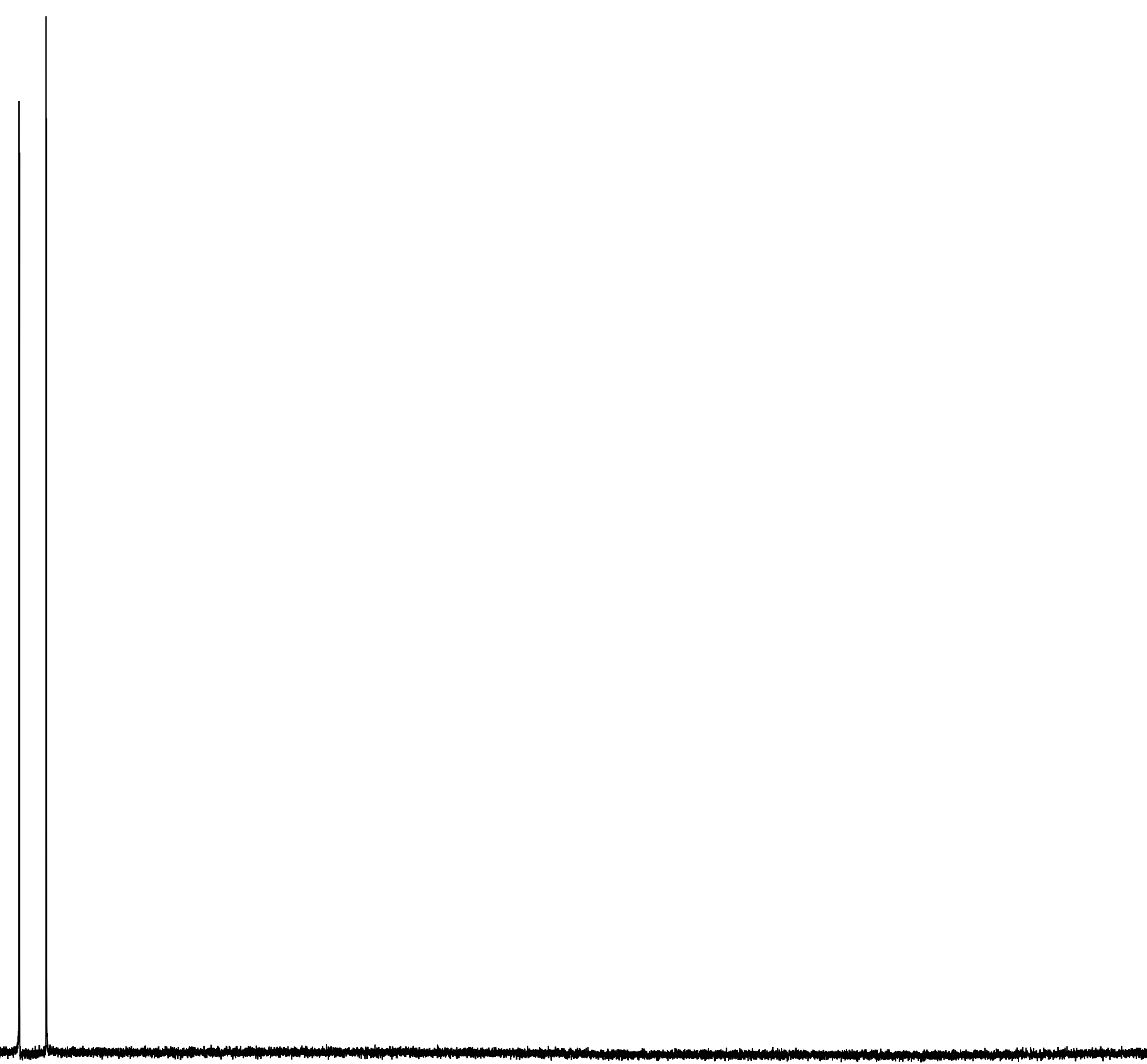




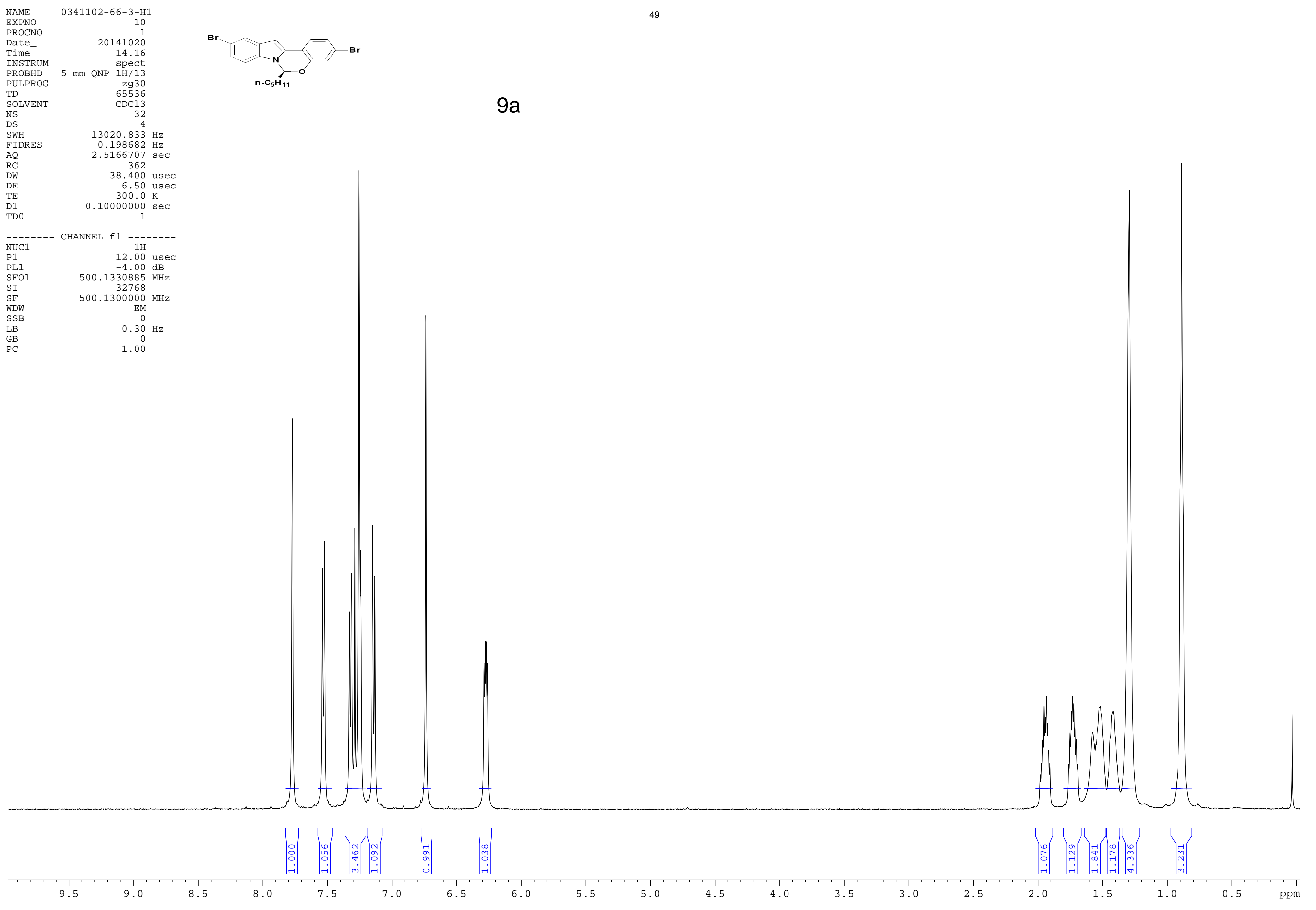




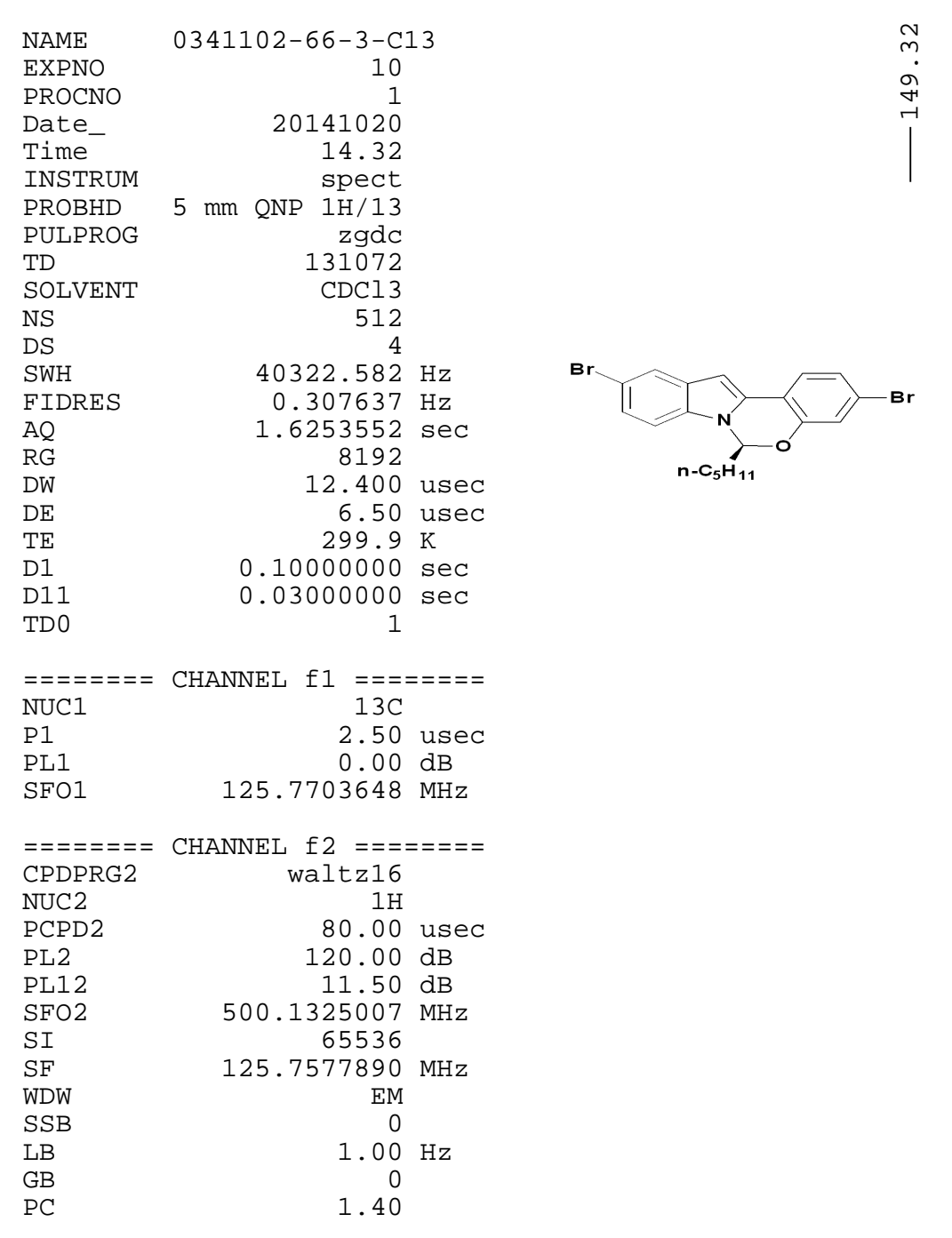




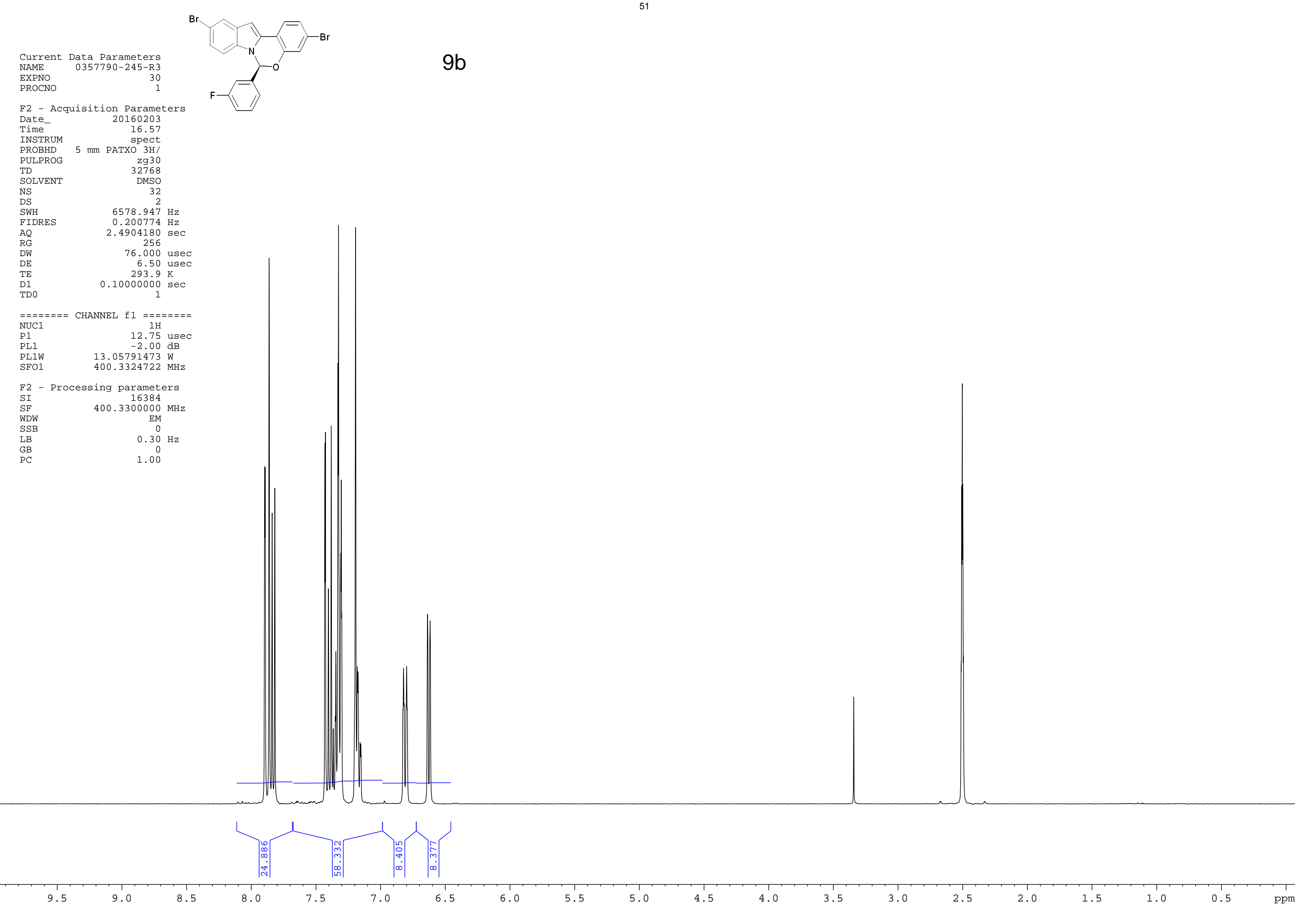


$\begin{array}{rr}\text { Current Data Parameters } \\ \text { NAME } & \text { O357790-176 }\end{array}$

EXPNO

F2 - Acquisition Parameters

Date

20160205
5.29

INSTRUM

$5 \mathrm{~mm}$ QNP $1 \mathrm{H} / 13$

TD

SOLVENT

131072

SWH $\quad 141843.969 \mathrm{~Hz}$

AQ $\quad \begin{array}{ll}1.082184 \mathrm{HZ} \\ \mathrm{AQ}\end{array}$

RG

$\begin{array}{lr}\text { DW } & 3.525 \text { usec } \\ \text { DE } & 5.04 \text { usec }\end{array}$

D1 $0.10000000 \mathrm{sec}$

$\begin{array}{ll}\text { D11 } & 0.03000000 \mathrm{sec} \\ \text { D12 } & 0.00002000 \mathrm{sec}\end{array}$

$========$ CHANNEL $f 1=======$
NUC1
$19 \mathrm{~F}$

$\begin{array}{lr}\text { NUC1 } & 19 F \\ \text { P1 } & 5.00 \text { usec } \\ \text { PL1 } & -4.00 \mathrm{~dB} \\ \text { SF01 } & 470.5217881 \mathrm{MHZ}\end{array}$

$========$ CHANNEL $\mathrm{f} 2========$
waltz16

$\begin{array}{lr}\text { NUC2 } & 1 \mathrm{H} \\ \text { PCPD2 } & 85.00 \text { usec } \\ \text { PL2 } & 120.00 \mathrm{~dB}\end{array}$

$12.00 \mathrm{~dB}$
$132500 \mathrm{~dB}$



F2 - Processing parameters

$\begin{array}{lr}\text { SI } & 65536 \\ \mathrm{SF} & 470.5923770 \mathrm{MHZ}\end{array}$

$\begin{array}{lr}\text { SF } & 470.5923770 \\ \text { WDW } & \text { MHz } \\ \text { SSB } & 0\end{array}$

$\begin{array}{lc}\text { SSB } & 0 \\ \mathrm{LB} & 1.00 \mathrm{~Hz} \\ \mathrm{~GB} & 0 \\ \mathrm{PC} & 1.00\end{array}$ 


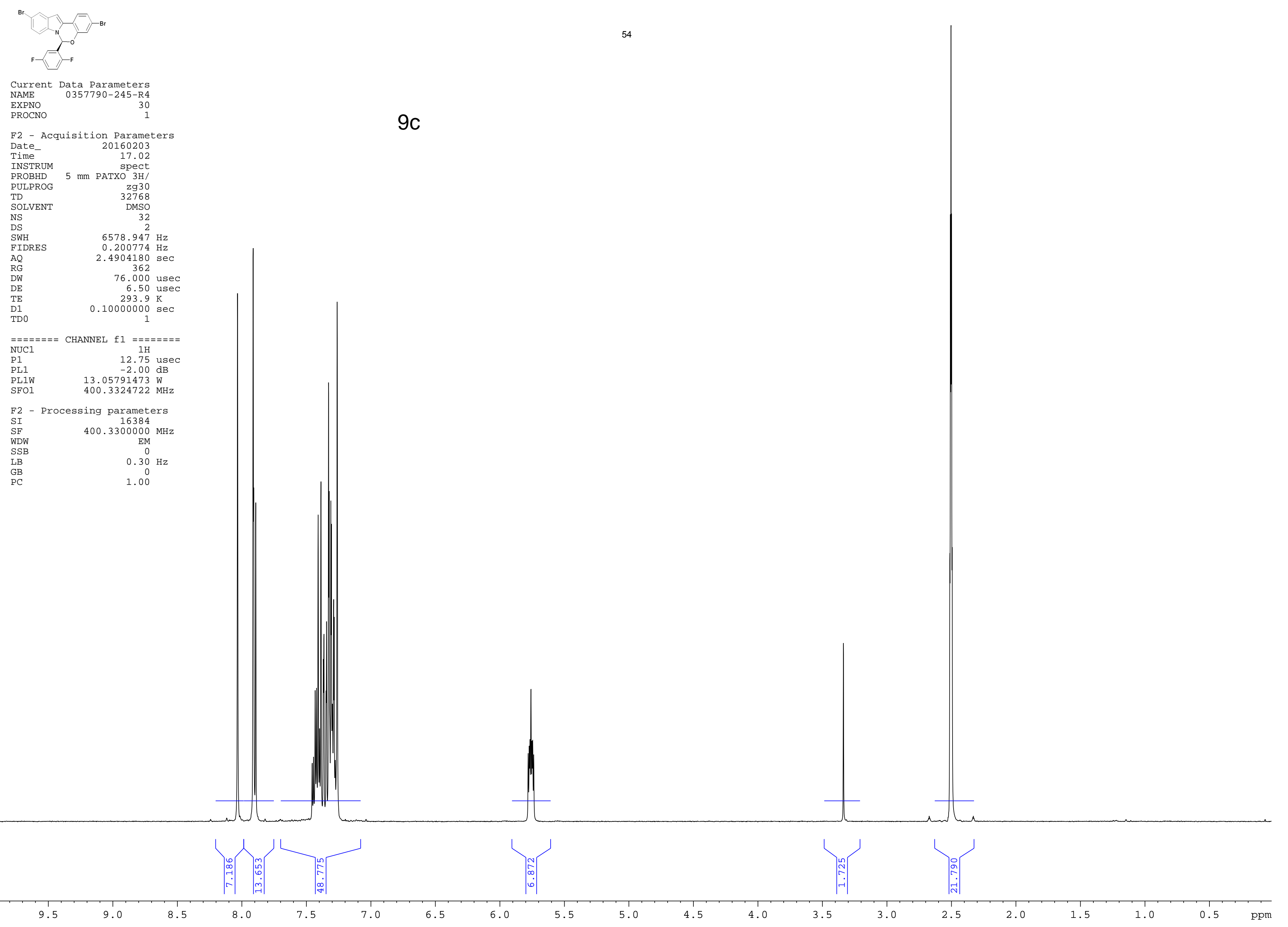




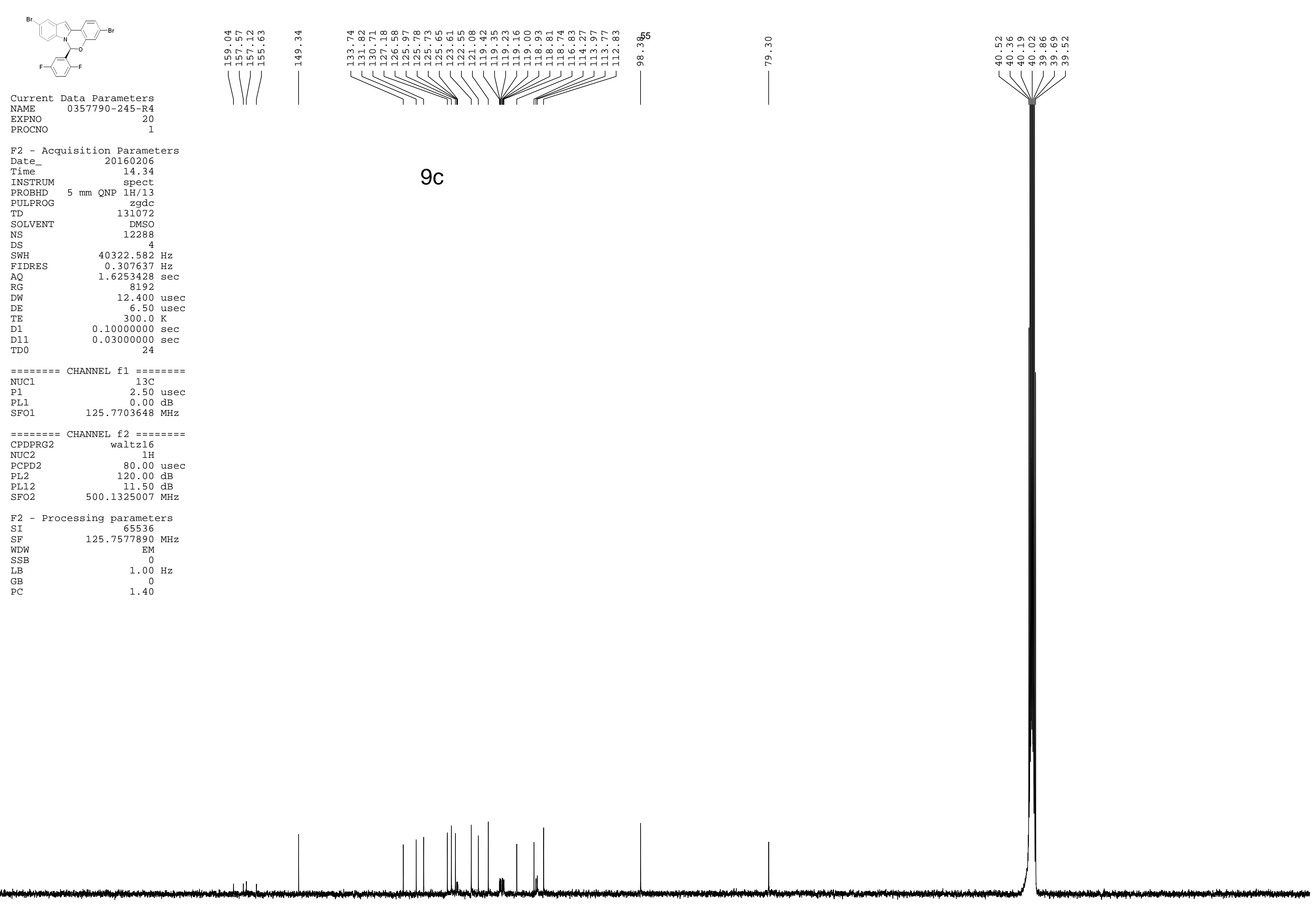



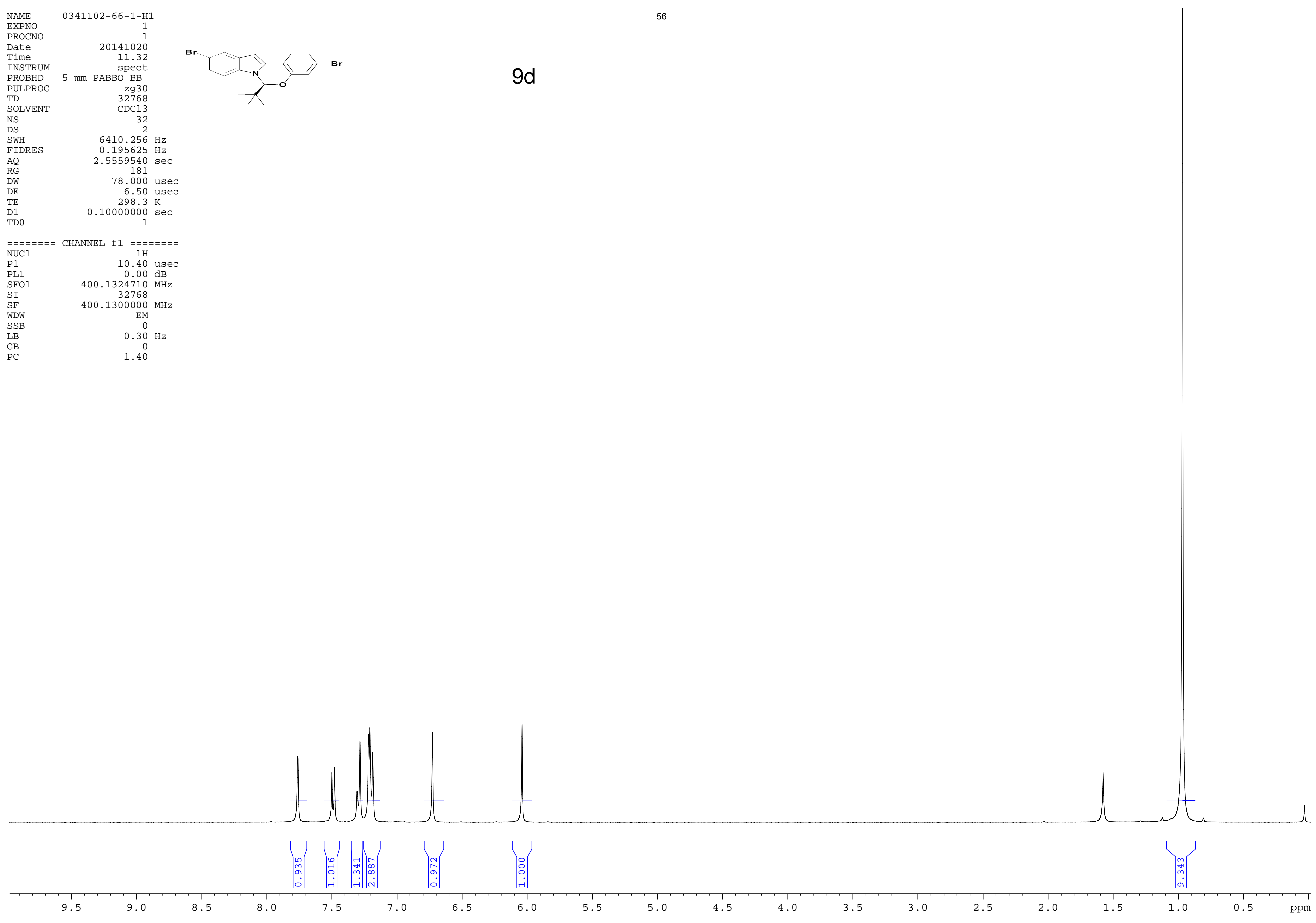


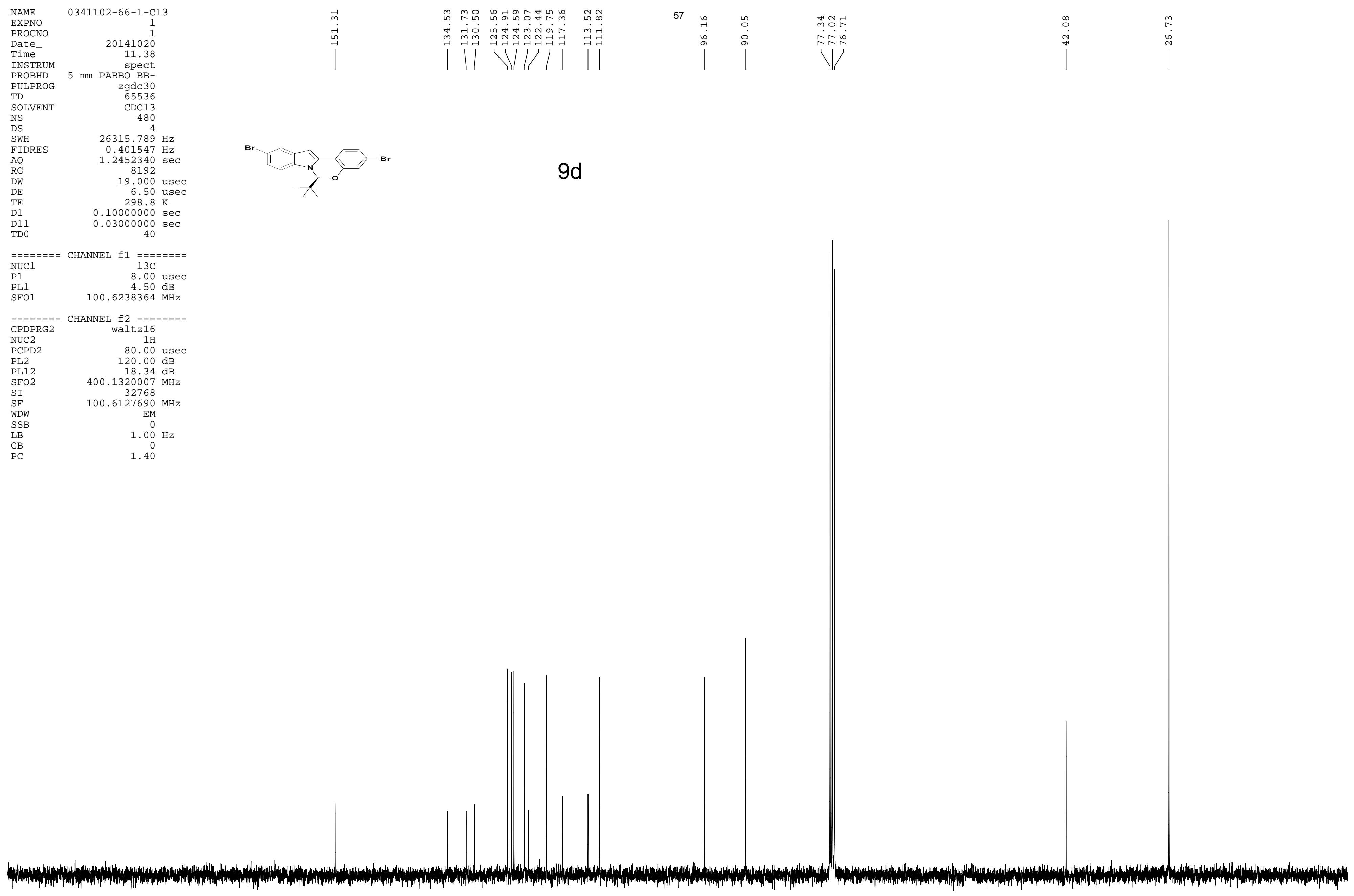




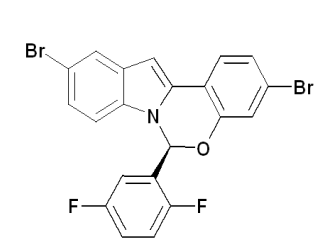

Current Data Parameters

EXPNO

F2 - Acquisition Parameters

Date

$\begin{array}{r}\text { Paramet } \\ 5.41 \\ \hline 16205\end{array}$

INTRUM
PROBHD

PULPROG

SOLVENT

$\begin{array}{lr}\text { DS } & 128 \\ \text { SWH } & 141843.969 \mathrm{~Hz} \\ \text { FIDRES } & 1.082184 \mathrm{~Hz}\end{array}$

$\begin{array}{ll}\mathrm{AQ} & 0.4620788 \\ \mathrm{RG} & \end{array}$

64
3.525 usec
5.04 usec

$300.1 \mathrm{~K}$

$\begin{array}{ll}\text { DE } & 0.100000000 \mathrm{sec} \\ \text { D1 } & 0.03000000 \mathrm{sec} \\ \text { D11 } & \end{array}$

$0.00002000 \mathrm{sec}$

$=======$ CHANNEL $\mathrm{f} 1 \mathrm{=}=======$
NUC1 $19 \mathrm{~F}$

$\begin{array}{lr}\text { P1 } & 5.00 \text { usec } \\ \text { PL1 } & -4.00 \mathrm{~dB}\end{array}$

SF01 470.52178

$=======$ CHANNEL $\mathrm{f} 2=====$
waltz16

NUC2
PCPD2

$\begin{array}{lr}\text { PL2 } & 85.00 \text { usec } \\ \text { PL12 } & 120.00 \mathrm{~dB}\end{array}$

$\begin{array}{lr}\text { PL12 } & 12.00 \mathrm{~dB} \\ \text { SF02 } & 500.1325007 \mathrm{MHz}\end{array}$

F2 - Processing parameters

$\begin{array}{lr}\text { SI } & 6556 \\ \mathrm{SF} & 470.5923770 \mathrm{MHz}\end{array}$

WDW $\quad 470.592377$ EM

$\begin{array}{lc}\text { SSB } & 0 \\ \mathrm{LB} & 1.00 \mathrm{~Hz} \\ \mathrm{~GB} & 0 \\ \mathrm{PC} & 1.00\end{array}$ 